\title{
The return to the hard state of GX 339-4 as seen by Suzaku
}

\author{
P.-O. Petrucci ${ }^{1}$, C. Cabanac ${ }^{2}$, S. Corbel ${ }^{3}$, E. Koerding ${ }^{4}$, and R. Fender ${ }^{5,6}$ \\ 1 UJF-Grenoble 1/CNRS-INSU, Institut de Planétologie et d'Astrophysique de Grenoble (IPAG) UMR 5274, 38041 Grenoble, France \\ e-mail: pierre-olivier.petrucci@obs.ujf-grenoble.fr \\ 2 Université de Toulouse, UPS-OMP, IRAP, Toulouse, France CNRS, IRAP, 9 Av du Colonel Roche, BP 44346, 31028 Toulouse \\ Cedex 4, France \\ ${ }^{3}$ Laboratoire AIM (CEA/IRFU - CNRS/INSU - Université Paris Diderot), CEA DSM/IRFU/SAp, 91191 Gif-sur-Yvette, France \\ ${ }^{4}$ Department of Astrophysics/IMAPP, Radboud University Nijmegen, PO Box 9010, 6500 GL Nijmegen, The Netherlands \\ 5 University of Oxford, Department of Physics, Astrophysics, Denys Wilkinson Building, Keble Road, OX1 3RH Oxford, UK \\ ${ }^{6}$ School of Physics and Astronomy, University of Southampton, Highfield, Southampton SO17 1BJ, UK
}

Received 12 July 2013 / Accepted 1 February 2014

\begin{abstract}
Disk recession is of prime importance to understand the physics of the state transitions in X-ray binaries. The microquasar GX 339-4 was observed by Suzaku five times spaced by a few days during its transition back to the hard state at the end of its 2010-2011 outburst. The $2-10 \mathrm{keV}$ source flux decreases by a factor $\sim 10$ between the beginning and the end of the monitoring. Simultaneous radio and optical/infrared (OIR) observations highlighted the re-ignition of the radio emission just before the beginning of the campaign with the maximum radio emission being reached between the first two Suzaku pointings, while the IR peaked a few weeks later. A fluorescent iron line is always significantly detected. Fits with Gaussian or Laor profiles give statistically equivalent results. In the case of a Laor profile, fits of the five data sets simultaneously agree with a disk inclination angle of $\sim 20$ degrees. The disk inner radius is $<10-30 R_{\mathrm{g}}$ in the first two observations but almost unconstrained in the last three due to the lower statistics. A soft X-ray excess is also present in these first two observations. Fits with a multicolored disk component give disk inner radii in qualitative agreement with those obtained with the iron line fits. The use of a physically more realistic model, including a blurred reflection component and a comptonization continuum, give some hints of the increase in the disk inner radius but the significances are always weak (and model dependent), preventing any clear conclusion concerning disk recession during this campaign. Interestingly, the addition of warm absorption significantly improves the fit of OBS1, while it is not needed in the other observations. Given the radio-jet re-ignition that occurs between OBS1 and OBS2, these absorption features may indicate the natural evolution of the accretion outflows transiting from a disk wind, which is a ubiquitous characteristic of soft states, and a jet, which is a signature of hard states. The comparison to a long 2008 Suzaku observation of GX 339-4 in a persistent faint hard state (similar in flux to OBS5), where a narrow iron line clearly indicates a disk recession, is discussed.
\end{abstract}

Key words. X-rays: binaries - X-rays: individuals: GX 339-4 - radiation mechanisms: general - accretion, accretion disks black hole physics

\section{Introduction}

Multiwavelength observation campaigns of microquasars, such as those done in X-ray and radio in the last 10 years, were crucial in bringing to light the striking link between the ejection phenomena (mostly observed in radio) and the inner accretion flow, whose radiation seems to be the dominant component in the X-rays (e.g., Corbel et al. 2000, 2003; Coriat et al. 2011; Gallo et al. 2003). For instance, strong radio emission, as interpreted by the presence of persistent jets (directly observed in a few cases, e.g., Dhawan et al. 2000; Stirling et al. 2001), is generally detected when the X-ray emission peaks at a few tens of keV in the so-called hard state (e.g., Corbel et al. 2004; Fender et al. 2004). This X-ray emission is commonly believed to originate via inverse Compton process from a plasma of hot electrons (the so-called corona) that scatters off UV/soft X-ray photons produced by the cooler part of the accretion flow. On the other hand the radio emission is quenched in the so-called soft state (Fender et al. 1999; Corbel et al. 2000), and the X-ray data are spectrally dominated by soft X-ray emission. This emission is generally interpreted as signature of a multicolored accretion disk component down to the last stable orbit $R_{\mathrm{ISCO}}$.
In the past ten years, high energy resolution observations of several microquasars also showed the presence of highly ionized absorption features in their X-ray spectra. These features were interpreted as a signature of ionized gas in the close environment of the black hole, their blueshifts indicating of outflows or winds (e.g., Miller et al. 2004 hereafter M04; Miller et al. 2006c). It has been realized that these features were more specifically observed in the soft state (e.g., Ponti et al. 2012; Diaz Trigo et al. 2011; Diaz Trigo \& Boirin 2013). These results suggest that X-ray binaries during outbursts may transit back and forth between diskjet (in the hard state) and disk-wind (in the soft state) configurations (e.g., Neilsen \& Lee 2009). However, the exact interplay between ejection and accretion phenomena and the origin of the transition from one state to the others is still poorly known (see, however, the recent study done by Kalemci et al. 2013.).

The commonly adopted toy-picture of the central regions of microquasars plays on the relative importance of the accretion disk and hot corona emission along the outburst (e.g., Esin et al. 1997; Done et al. 2007). The accretion disk is assumed to be present in between an outer and inner radius, $R_{\text {out }}$ and $R_{\text {in }}$, while the hot corona is localized in between $R_{\text {in }}$ and $R_{\mathrm{ISCO}}$. In the hard state, $R_{\mathrm{in}} \gg R_{\mathrm{ISCO}}$, and the hot corona dominates the observed 
Table 1. Log of the five observations.

\begin{tabular}{|c|c|c|c|c|c|c|c|}
\hline Obs. name & Obs. ID & MJD & $\begin{array}{l}\text { XIS03 Exp. } \\
\text { ks }\end{array}$ & $\begin{array}{r}\text { XIS 0-3 } \\
0.7-2 \mathrm{keV}\left(\mathrm{s}^{-1}\right) \\
\end{array}$ & $\begin{array}{r}\text { XIS 0-3 } \\
2-10 \mathrm{keV}\left(\mathrm{s}^{-1}\right) \\
\end{array}$ & $\begin{array}{c}\text { HXD/PIN } \\
20-70 \mathrm{keV}\left(\mathrm{s}^{-1}\right)\end{array}$ & $\begin{array}{c}\mathrm{HXD} / \mathrm{GSO} \\
70-200 \mathrm{keV}\left(\mathrm{s}^{-1}\right)\end{array}$ \\
\hline OBS1 & 405063010 & 55603.7 & 44.2 & $11.90 \pm 0.02$ & $10.22 \pm 0.02$ & $0.62 \pm 0.01$ & $0.96 \pm 0.04$ \\
\hline OBS2 & 405063020 & 55608.9 & 42.0 & $5.20 \pm 0.01$ & $6.55 \pm 0.01$ & $0.46 \pm 0.01$ & $1.05 \pm 0.04$ \\
\hline OBS3 & 405063030 & 55616.8 & 38.4 & $1.81 \pm 0.01$ & $2.80 \pm 0.01$ & $0.20 \pm 0.01$ & $0.74 \pm 0.05$ \\
\hline OBS4 & 405063040 & 55620.2 & 43.6 & $1.32 \pm 0.01$ & $2.09 \pm 0.01$ & $0.15 \pm 0.01$ & $0.74 \pm 0.04$ \\
\hline OBS5 & 405063050 & 55627.5 & 37.3 & $0.92 \pm 0.01$ & $1.50 \pm 0.01$ & $0.10 \pm 0.01$ & $0.56 \pm 0.05$ \\
\hline
\end{tabular}

Notes. Are listed: their ID number, the corresponding date (in MJD), the sum of the XIS0 and XIS3 exposure time (each exposure being 20 ks, the total exposure of the XIS0 and XIS3 instruments is about twice this time), and the count rates in the different Suzaku instruments.

emission. The inner part of the accretion disk (close to $R_{\text {in }}$ ) is then cold which explains its poor detection in the soft X-rays in this state. In contrast, in the soft states $R_{\text {in }} \sim R_{\mathrm{ISCO}}$, i.e. the hot corona is no more present and the spectra are dominated by the accretion disk emission.

If this picture is correct, variations of the disk inner radius $R_{\text {in }}$ should occur during the state transitions, by first decreasing during the hard-to-soft transition but then increasing during the softto-hard one. This interpretation is apparently supported by the observations in hard states of weak reflection components (e.g., Gierlinski et al. 1997; Barret et al. 2000; Miller et al. 2002; Zycki et al. 1998; Joinet et al. 2007), the absence of relativistic broadening of the iron line in a few cases (e.g., Tomsick et al. 2009; Plant et al. 2013), and the absence of obvious thermal components (e.g., Poutanen et al. 1997; Dove et al. 1997; Remillard \& McClintock 2006; Done et al. 2007; Dunn et al. 2010), which are all potential signatures of small and remote reflecting area.

The variable disk inner radius during state transitions then becomes a natural key ingredient in most theoretical models, which controls or results from the spectral and timing evolution of microquasars during the outburst (e.g., Esin et al. 1997; Meyer et al. 2000; Belloni et al. 2005; Remillard \& McClintock 2006; Ferreira et al. 2006; Petrucci et al. 2008).

Recent XMM observations, which are much more sensitive, especially in the soft X-rays (i.e. below $2 \mathrm{keV}$ ), when compared to the other existing X-ray missions, apparently ruled out the presence of a recessed disk in the hard states (e.g., Miller et al. 2006a,b). The long XMM observation monitored during the 2004 outburst of GX 339-4 did not agree with a simple powerlaw although the system was in a typical, although bright $\left(\sim 10 \% L_{\text {Edd }}\right)$, hard state. According to the authors, a very strong soft excess and a broad emission feature around $6.4 \mathrm{keV}$ were also present and well fitted by a multicolored disk and a relativistically broadened emission line, respectively. This suggests the presence of an accretion disk extending towards the vicinity of the black hole (see below). The Swift/XRT observations of XTE J1817-330, during its decline to the hard state, led Rykoff et al. (2007) to the same conclusions, that is no disk recession. From their Swift survey of stellar mass black holes, Reynolds \& Miller (2013) do not find evidence for large-scale truncation of the accretion disk in the hard state for at least X-ray luminosities larger than $10^{-3} L_{\text {Edd }}$.

The estimates of inner disk radii based on continuum spectroscopy are subject however to considerable uncertainties (e.g., Merloni et al. 2000; Zimmerman et al. 2005; Cabanac et al. 2009 hereafter (09) and a different data analysis may give different conclusions. A recent re-analysis of the data used in Miller et al. (2006b) suggests that the observed broad iron line may be an artifact due to an improper correction of the pile-up in the MOS data (Done \& Diaz Trigo 2010). Suzaku, who points on the same source in its hard state, has also shown that the spectrum could be consistent with a truncated disk (Tomsick et al. 2009). More specifically, by re-analyzing the whole Swift/XRT dataset of XTE J1817-330, C09 does observe a slight increase of the disk radius. This apparently occurs when the $2-10 \mathrm{keV}$ luminosity decreases below $\sim 5 \times 10^{-3} L_{\text {Edd }}{ }^{1}$. These authors analyzed other sources in the same way and obtain similar, though less significant, results.

The reality of the disk recession is clearly of prime importance in that we crucially need it if we want to understand the physics of the state transitions. By confirming, and precisely measuring, this recession (if any) should constrain and refine most of the present theoretical models. On the other hand, the absence of recession implies that we strongly reconsider our present understanding of the microquasar phenomenon.

In this paper we present a Suzaku campaign on the microquasar GX 339-4 that aims at catching any recession of the accretion disk during a soft-to-hard state transition. Section 2 detailed the observation and data treatment and Sect. 3 the data analysis. While the constraints on the disk inner radius, as discussed in Sect. 4, prevent any clear conclusions concerning its recession, the observation of ionized absorbing features in the soft X-rays may suggest a disk wind, whose properties may evolve during the transitions. Theses results are discussed in Sect. 5 before concluding.

\section{Observations and data treatment}

\subsection{Suzaku observations}

GX 339-4 was observed five times ( $20 \mathrm{ks}$ each) by Suzaku at the end of its last outburst in February 2011, as soon as the source became visible by the satellite. The five observations were separated by a few days to follow the spectral evolution of the object along all its transition back to the hard state. The log of these observations is detailed in Table 1 with the corresponding dates. The RXTE/PCA 3-20 keV light curve of the complete 2010-2011 outburst of GX 339-4 is plotted at the top of Fig. 1 with the hardness ratio ${ }^{2}$. A zoom of the last part of the outburst, with the dates of the five Suzaku observations as indicated by the vertical dotted lines, is shown at the bottom of Fig. 1.

For the data treatment, we use the most up-to-date calibration files and the HEASoft version 11.6.1. We run the Suzaku XIS/HXD aepipeline (V1.1.0) tools to reprocess the data from scratch. We consider the pile-up effect in the XIS instrument by first running the aeattcor 2 tool, which corrects the Suzaku attitude data for the effects of "thermal wobbling" caused by

\footnotetext{
1 Assuming a ten-solar-mass black hole.

2 The hardness ratio is defined as the ratio of the $(5.7-9.5 \mathrm{keV})$ count rate over the (2.9-5.7 keV) count rate.
} 

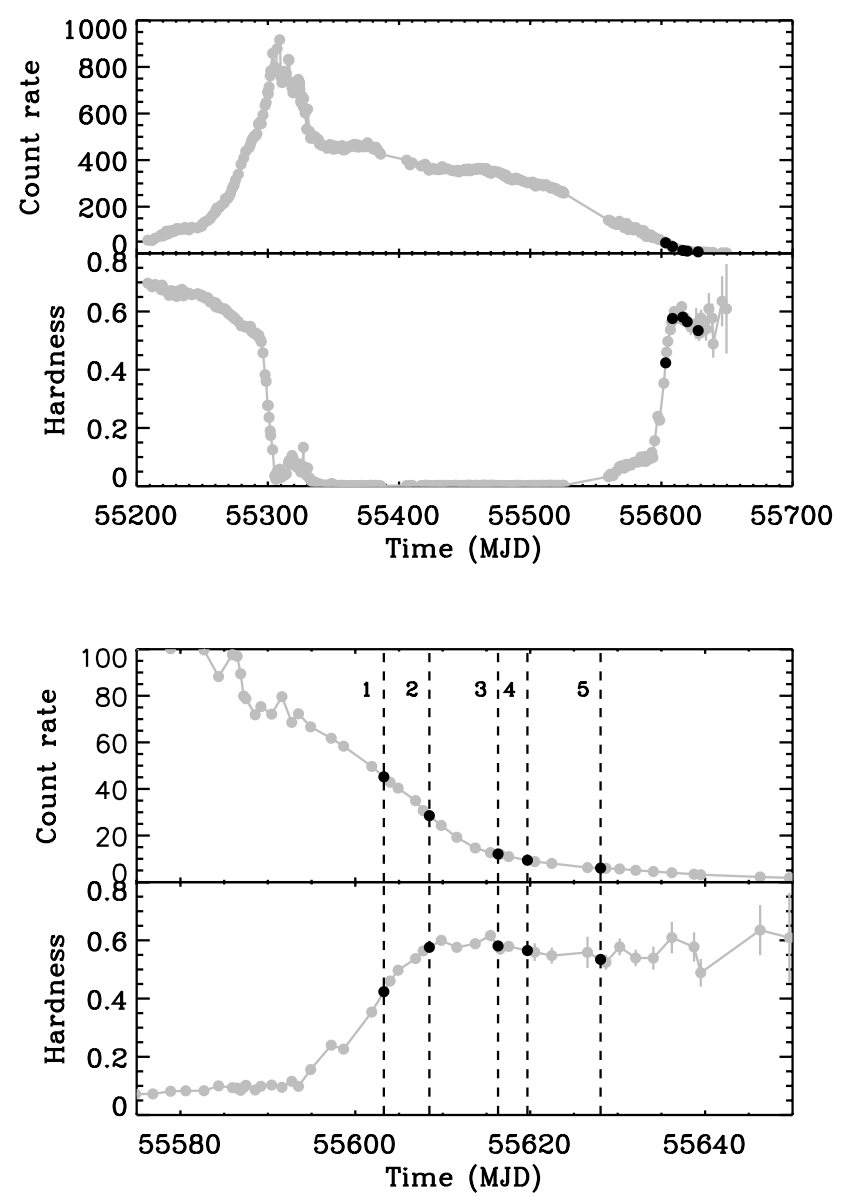

Fig. 1. Top: RXTE/PCA 3-20 keV count rate and hardness ratio light curves of the complete 2010-2011 outburst of GX 339-4. Bottom: a zoom at the end of the outburst, with the date of the five Suzaku observations indicated by the black dots and the vertical dotted lines.

thermal distortions of the satellite bodies ${ }^{3}$. Then, we run the pileup fraction estimation tool pileest which is also released in the HEASoft package to estimate the amount of pile up in the XIS images and disregarded regions with $>10 \%$ pile-up fraction during the spectral extraction. Only the first two observations suffered from pile-up ( $\sim 19$ and $10 \%$ for OBS1 and OBS2, respectively, for the most central pixels) and a circular region of $\sim 10^{\prime \prime}$ radius in the central part of the image was excluded. We added together the XISO and XIS3 spectra. Unless specified in the text, the XIS spectra were all rebinned so that the minimum number of bins per resolution element is five in order to ensure a minimum number of counts per channel ${ }^{4}$ of 30 .

To create the background files in the XIS energy range we use the ftool xisnxbgen which estimates the non X-ray background spectrum of the XIS instrument. While the cosmic X-ray background is expected to be low in the XIS energy band, we take it into account by following the recipe indicated in the ABC guide V4.0 (pp. 81-82) ( $^{5}$ but renormalized it to the XIS FOV that corresponds to the $1 / 4$ window mode. The total

\footnotetext{
3 http://heasarc.gsfc.nasa.gov/ftools/caldb/help/ aeattcor2.html

4 For the rebin procedure, we use the tool PHARBN developed by M. Guainazzi and adapted to the XIS instrument.

5 http://heasarc.gsfc.nasa.gov/docs/suzaku/analysis/ abc/
}

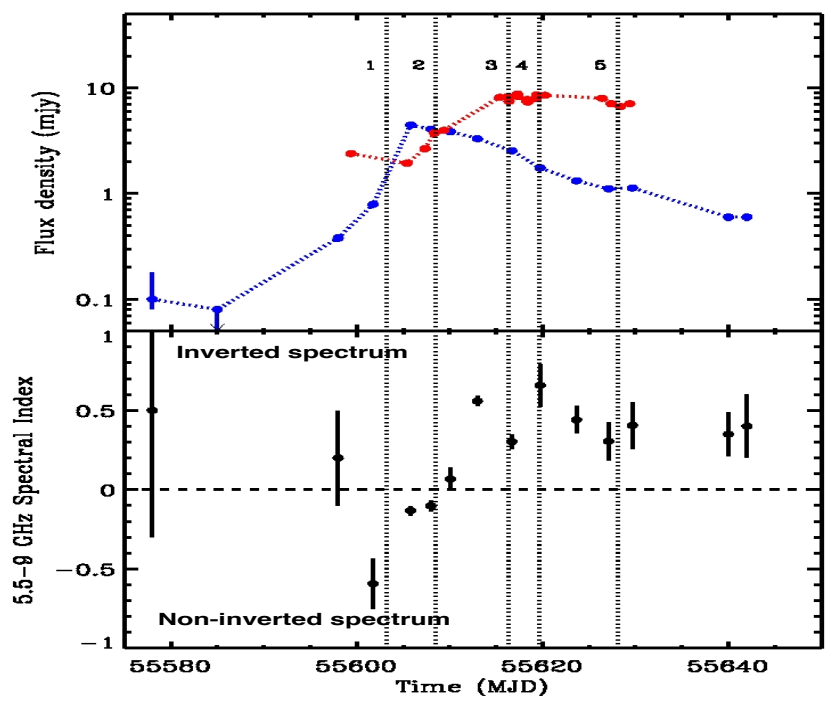

Fig. 2. Top: $5.5 \mathrm{GHz}$ (blue) and $H$ band (red) light curves. Bottom: light curve of the 5.5-9 GHz spectral index. A positive spectral index corresponds to an inverted spectrum that is generally characteristic of an optically thick synchrotron emission from a stratified jet (e.g., Blandford \& Königl 1979). The dates of the five Suzaku observations are indicated by the vertical dotted lines (data from Corbel et al. 2013).

X-ray background files (including the non-X-ray and the cosmic $\mathrm{X}$-ray background) of the HXD/PIN instruments were computed using the ftools hxdpinxbpi ${ }^{6}$. For the non X-ray background, we use the "tuned" background files distributed by the HXD team and corresponding to our observations. These "tuned" background suffers from systematic uncertainties of about $1.3 \%$ that have been added to the PIN data during our fitting procedure.

Following the ABC guide V.4.0 (Chap. 5.7.2), the normalization of the HXD/PIN with respect to XIS is fixed to 1.16. The energy ranges for the XIS and HXD/PIN instruments are restricted to $0.7-10 \mathrm{keV}$ and $20-70 \mathrm{keV}$ respectively. In the following, we name the five observations OBS1, OBS2, OBS3, OBS4, and OBS5 (see Table 1).

\subsection{Radio and IR observations}

Simultaneous or quasi-simultaneous radio observations were taken with the Australia Telescope Compact Array (ATCA) at 5.5 and $9 \mathrm{GHz}$ (Corbel et al. 2013, hereafter C13). Simultaneous or quasi-simultaneous optical and IR observations were taken with the SMARTS telescope (Buxton et al. 2012; Dinçer et al. 2012). The corresponding light curves are reported at the top of Fig. 2 and the radio spectral index (between the 5.5 and $9 \mathrm{GHz}$ band) is plotted at the bottom of the figure.

\section{Results}

\subsection{Light curves}

GX 339-4 was at the end of its outburst, and as expected, its X-ray flux decreases from OBS1 to OBS5. The count rates in the $0.7-2,2-10,20-70$ and $70-200 \mathrm{keV}$ ranges are indicated

\footnotetext{
6 We do not include the GSO data in the spectral analysis due to their very poor statistics.

7 See http://heasarc.gsfc.nasa.gov/docs/suzaku/ analysis/watchout.html, item 16.
} 


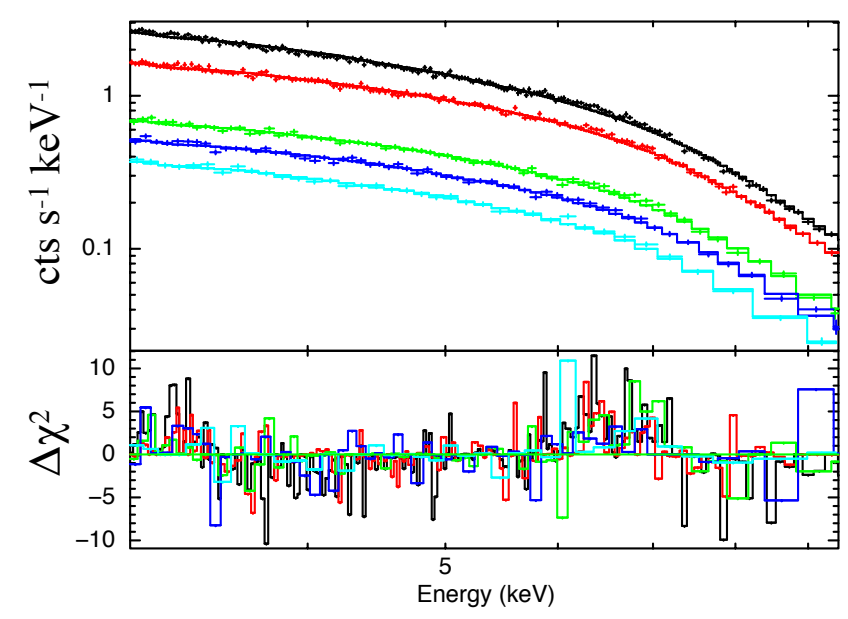

Fig. 3. Top panel: folded spectra of the five Suzaku observations (OBS1 to OBS5 from top to bottom) fitted by a power law in the $3-10 \mathrm{keV}$ energy range. For clarity, only the XISO+XIS3 spectra are plotted. They have been rebinned to have $30 \sigma$ per bin. The bottom panel shows the contributions to the $\chi^{2}$.

in Table 1, and indeed, they all decrease during the campaign. Interestingly, the decrease is much more pronounced in the softer energy range than in the harder ones. The $0.7-2 \mathrm{keV}$ range count rate decreases by more than a factor 10 , while it decreases by less than a factor 2 in the $70-200 \mathrm{keV}$ range. This clearly indicates a spectral change of the X-ray emission.

As shown in Fig. 2, the radio emission begins to increase between MJD 55 590-55 600 and reaches a maximum between our two first Suzaku observations ( MJD 55605 ). The radio emission is generally associated with the presence of a jet; the increase of radio flux is interpreted as the reappearance of the jet while GX 339-4 is turning back to its hard state (see e.g., C13; Kalemci et al. 2013). This is well supported by the simultaneous increase of the radio spectral index (bottom plot on Fig. 2), which becomes positive after OBS2. Thus inverted spectrum is characteristic of an optically thick synchrotron emission from a stratified jet (e.g., Blandford \& Königl 1979).

It is interesting to note that the RXTE/PCA hardness ratio (see Fig. 1) begins to increase a few days before the radio peak, the two events are clearly separated in time (see also Kalemci et al. 2013 for other outbursts). Concerning the $\mathrm{H}$ band emission, it reaches a local maximum about 10 days after the radio peak in between OBS3 and OBS4 (see discussion in C13).

\subsection{Spectral analysis}

\subsubsection{Above $3 \mathrm{keV}$ : XIS data alone}

We begin our spectral analysis on the Suzaku data above $3 \mathrm{keV}$, which is a spectral domain known to be dominated by the primary continuum. We first fit the XIS data only between 3 and $10 \mathrm{keV}$ with a simple power law model. The best-fit values for the photon index are reported in Table 2, and the folded spectra are plotted in Fig. 3. The photon index decreases rapidly between OBS1 and OBS2, from $\Gamma=1.72 \pm 0.01$ to $\Gamma=1.56 \pm 0.01$ and then stays roughly constant around 1.55 between OBS 2 and OBS5.

Excesses close to 6-7 keV are visible especially in OBS1, 2 and 3, suggesting the presence of an iron $\mathrm{K} \alpha$ line. We add a Gaussian component and fit the spectra again. The fit improves very significantly for OBS1 $\left(\Delta \chi^{2}=143\right)$, which is less for OBS2 and $3\left(\Delta \chi^{2}=29\right.$ and 30 respectively) and even less for OBS4 and $5\left(\Delta \chi^{2}=14\right)$ but the improvement is always significant (at more than $99 \%$ following the F-test ${ }^{8}$ ). The best-fit parameter values are reported in Table 2 . The contour plots of the line flux vs. line energy and line width vs. line energy for the five observations are plotted in Fig. 4.

The line flux is clearly decreasing between OBS1 and OBS2 (at more than $3 \sigma$ ). Then it is consistent with a constant at a confidence level of $\sim 70 \%$ between OBS2 and OBS5. Concerning the line energy, its highest value is reached in OBS3 with $E_{\text {gauss }}=$ $6.77 \pm 0.13$. The other measurements are consistent with a neutral iron line peaking at $6.4 \mathrm{keV}$. The significance of the variability in the line energy during the campaign is however less than $40 \%$. The line width is consistent with a constant at a confidence level of $\sim 60 \%$. It has an average value of $\sim 440 \pm 70 \mathrm{eV}$ which potentially indicates some broadening.

To test if the line broadening could be due to relativistic effects, we replace the Gaussian profile by a Laor profile, which is expected if the line emission is produced by an accretion disk around a black hole. To limit the number of free parameters, we fix the outer radius of the disk $R_{\text {out }}$ to $400 R_{\mathrm{g}}$ and the index of the radial power law dependence of the disk emissivity to 3 . We then fit the five XIS spectra simultaneously, only imposing the same disk inclination angle (which was let free to vary) for the five observations. All the other parameters (Laor model inner radius and normalization, power law photon index and normalization) were independent from one observation to the other and free to vary. The fit gives a best-fit value of the inclination angle $i=21 \pm 7 \mathrm{deg}$, which is consistent with past measurements (e.g., M04, Reis et al. 2008). The best-fit values of the other parameters are reported in Table 2 and the contour plots of the disk inclination vs. the disk inner radius are reported in Fig. 5.

The fits are statistically as good as with a Gaussian profile; the line flux and equivalent width behaviors are completely consistent between the two profiles. Compared to the Gaussian fits, the line energy is now consistent with a constant between the five observations and it is significantly higher with a mean value $\left\langle E_{\text {Laor }}\right\rangle=6.96 \pm 0.07 \mathrm{keV}$. Concerning the disk inner radius, it is well constrained to a few Schwarzschild radii for OBS1 and OBS2, while we have only upper limits for the three last observations. While it strongly suggests the presence of the accretion disk very close to the black hole at the beginning of the campaign, the data prevent any clear conclusion concerning the disk recession.

\subsection{2. $0.7-10 \mathrm{keV}$ : XIS data alone}

We now include the $0.7-3 \mathrm{keV}$ data in the fitting procedure. The data/model ratios of the five XIS spectra, which are obtained after extrapolation of the best-fit POWER LAW + LAOR model down to $0.7 \mathrm{keV}$, are plotted in Fig. 6. A photo-electric absorption (model TBNEW $^{9}$ in XSPEC) was added but the hydrogen column density was fixed to $6 \times 10^{21} \mathrm{~cm}^{2}$, which is the typical galactic $N_{H}$ observed in the direction of GX 339-4 (e.g., Zdziarski et al. 2004; Cadolle Bel et al. 2011). The soft X-ray part of the spectra varies in time, in excess above the power law extrapolation in OBS1 and OBS2, but in deficit in the three last observations. Letting the column density free to vary improves the fits, but they are

\footnotetext{
8 See however Protassov et al. (2002) about the usage of the F-test in line-like features.

9 All modeling was performed using the "wilm" abundances (Wilms et al. 2000) with the vern cross-sections (Verner et al. 1996).
} 
Table 2. Best fit of the XIS data between 3 and $10 \mathrm{keV}$ with a simple POWER LAW, a POWER-LAW + GAUSSIAN line and a POWER-LAW + LAOR Profile emission line.

\begin{tabular}{|c|c|c|c|c|c|c|}
\hline Obs. & $\Gamma$ & $\begin{array}{c}F_{3-10 \mathrm{keV}} \\
\left(\times 10^{-10} \mathrm{erg} \mathrm{cm}^{-2} \mathrm{~s}^{-1}\right)\end{array}$ & $\begin{array}{l}\text { Power law } \\
L_{3-10 \mathrm{keV}} / L_{\mathrm{Edd}} \\
(\%)\end{array}$ & & & $\chi^{2} /$ d.o.f. \\
\hline 1 & $1.72 \pm 0.01$ & 3.35 & 0.188 & & & $429 / 254$ \\
\hline 2 & $1.56 \pm 0.01$ & 2.05 & 0.115 & & & $303 / 254$ \\
\hline 3 & $1.53 \pm 0.02$ & 0.89 & 0.050 & & & $297 / 251$ \\
\hline 4 & $1.53 \pm 0.02$ & 0.66 & 0.037 & & & $314 / 245$ \\
\hline 5 & $1.57 \pm 0.02$ & 0.47 & 0.026 & & & $220 / 219$ \\
\hline 2008 & $1.52 \pm 0.01$ & 0.44 & 0.024 & & & $435 / 254$ \\
\hline Obs. & $\Gamma$ & $\begin{array}{l}E_{\text {gauss }} \\
(\mathrm{keV})\end{array}$ & $\begin{array}{c}\text { wer law+Gauss } \\
\begin{array}{c}\sigma_{\text {gauss }} \\
(\mathrm{keV})\end{array}\end{array}$ & $\begin{array}{c}F_{\text {gauss }} \\
\left(\times 10^{-4} \mathrm{ph} \mathrm{cm}^{-2} \mathrm{~s}^{-1}\right)\end{array}$ & $\begin{array}{l}E W \\
(\mathrm{eV})\end{array}$ & $\chi^{2} /$ d.o.f. \\
\hline 1 & $1.81 \pm 0.02$ & $6.40 \pm 0.10$ & $0.66 \pm 0.13$ & $6.5_{-1.4}^{+1.7}$ & $150_{-35}^{+40}$ & $285 / 251$ \\
\hline 2 & $1.60 \pm 0.02$ & $6.49 \pm 0.12$ & $0.35 \pm 0.13$ & $1.5_{-0.5}^{+0.6}$ & $55_{-20}^{-25}$ & $267 / 251$ \\
\hline 3 & $1.57 \pm 0.02$ & $6.77 \pm 0.13$ & $0.36 \pm 0.11$ & $1.0_{-0.4}^{+0.5}$ & $90_{-35}^{+40}$ & $263 / 248$ \\
\hline 4 & $1.56 \pm 0.03$ & $6.42 \pm 0.20$ & $0.36 \pm 0.22$ & $0.5_{-0.3}^{-0.4}$ & $60_{-35}^{+355}$ & $300 / 242$ \\
\hline 5 & $1.62 \pm 0.06$ & $6.59 \pm 0.30$ & $0.60 \pm 0.51$ & $0.7_{-0.4}^{-1.3}$ & $130_{-105}^{+75}$ & $207 / 216$ \\
\hline 2008 & $1.54 \pm 0.01$ & $6.41 \pm 0.02$ & $0.10 \pm 0.03$ & $0.4_{-0.1}^{+0.1}$ & $65_{-10}^{+10}$ & $248 / 251$ \\
\hline Obs. & $\Gamma$ & $\begin{array}{c}E_{\text {laor }} \\
(\mathrm{keV})\end{array}$ & $\begin{array}{c}\text { wer law }+ \text { Laor } \\
R_{\mathrm{in}} \\
\left(R_{\mathrm{g}}\right)\end{array}$ & $\begin{array}{c}F_{\text {laor }} \\
\left(\times 10^{-4} \mathrm{ph} \mathrm{cm}^{-2} \mathrm{~s}^{-1}\right)\end{array}$ & $\begin{array}{l}E W \\
(\mathrm{eV})\end{array}$ & $\chi^{2} /$ d.o.f. \\
\hline 1 & $1.80_{-0.01}^{+0.02}$ & $7.00_{-0.08}^{+0.13}$ & $5.0_{-0.6}^{+0.5}$ & $6.0_{-0.9}^{+1.1}$ & $160_{-30}^{+40}$ & $286 / 250$ \\
\hline 2 & $1.60_{-0.02}^{+0.01}$ & $6.84_{-0.14}^{+0.08}$ & $6.3_{-1.3}^{+2.6}$ & $1.9_{-0.3}^{-0.9}$ & $80_{-30}^{+30}$ & $266 / 250$ \\
\hline 3 & $1.58_{-0.03}^{+0.02}$ & $7.06_{-0.013}^{+0.14}$ & $<35$ & $1.3_{-0.7}^{+0.5}$ & $140_{-60}^{+60}$ & $260 / 247$ \\
\hline 4 & $1.56_{-0.03}^{+0.03}$ & $6.82_{-0.48}^{+0.132}$ & $<180$ & $0.7_{-0.4}^{-0.7}$ & $90_{-70}^{+60}$ & $300 / 241$ \\
\hline 5 & $1.61_{-0.02}^{-0.06}$ & $7.03_{-0.19}^{+0.38}$ & $<25$ & $0.7_{-0.2}^{-0.4}$ & $120_{-90}^{+130}$ & $207 / 215$ \\
\hline 2008 & $1.54_{-0.01}^{+0.01}$ & $6.43_{-0.02}^{+0.02}$ & $>100$ & $0.38_{-0.04}^{+0.05}$ & $65_{-5}^{+5}$ & $248 / 251$ \\
\hline
\end{tabular}

Notes. In the last case, the five XIS spectra have been fitted simultaneously, letting all the parameters free to vary but imposing the LAOR profile inclination angle to be the same between the five models. The best-fit value for the inclination angle is $i=21_{-7}^{+5}$ deg. The errors on the inner disk radius $R_{\text {in }}$ of the LAOR profile correspond to a confidence level of $90 \%$ for two parameters. We have also reported the best fit parameter values for the 2008 Suzaku observation, again assuming the same inclination angle as the five other Suzaku observations. With the addition of these data, the best-fit value for the inclination angle then becomes $i=20_{-6}^{+3}$ deg. To compute the $3-10 \mathrm{keV}$ flux in Eddington units, we adopt an Eddington luminosity of $L_{\mathrm{Edd}} \simeq 1.3 \times 10^{39} \mathrm{erg} \mathrm{s}^{-1}$ (i.e. we assume a ten-solar-mass black hole) and a distance to GX $339-4$ of $8 \mathrm{kpc}$.

Table 3. Best fit of the XIS data between 0.7 and $10 \mathrm{keV}$ with a DISKBB + POWER-LAW + LAOR line and a photo-electric absorption (model TBNEW, Wilms et al. 2000).

\begin{tabular}{|c|c|c|c|c|c|c|c|c|c|c|}
\hline \multirow[b]{2}{*}{ Obs. } & \multicolumn{9}{|c|}{ tbnew $*($ diskbb+power law+laor $)$} & \multirow[b]{2}{*}{$\Delta \chi^{2}$} \\
\hline & $\begin{array}{r}N_{\mathrm{h}} \\
\times 10^{22} \\
\end{array}$ & $\begin{array}{c}T_{\text {in }} \\
(\mathrm{eV})\end{array}$ & $\begin{array}{l}N_{\text {diskbb }} \\
\times 10^{3}\end{array}$ & $\Gamma$ & $\begin{array}{c}E_{\text {Laor }} \\
(\mathrm{keV})\end{array}$ & $\begin{array}{c}R_{\text {in,Laor }} \\
\left(R_{\mathrm{g}}\right)\end{array}$ & $\begin{array}{c}F_{\text {Laor }} \\
\times 10^{-4} \\
\end{array}$ & $\begin{array}{l}E W \\
(\mathrm{eV})\end{array}$ & $\chi^{2} /$ d.o.f. & \\
\hline 1 & $0.47_{-0.01}^{+0.01}$ & $330_{-10}^{+10}$ & $1.0_{-0.2}^{+0.2}$ & $1.84_{-0.01}^{+0.02}$ & $7.04_{-0.07}^{+0.06}$ & $5.0_{-0}^{+0.9}$ & $5.9_{-09}^{+1.0}$ & $160_{-30}^{+30}$ & $593 / 384$ & 1332 \\
\hline 2 & $0.63_{-0.03}^{+0.04}$ & $210_{-10}^{+10}$ & $5.3_{-1.9}^{+2.8}$ & $1.69_{-0.01}^{+0.01}$ & $6.89_{-0.09}^{+0.12}$ & $6.8_{-16}^{+2.3}$ & $2.1_{-05}^{+0.9}$ & $80_{-30}^{+30}$ & $441 / 384$ & 173 \\
\hline 3 & $0.57_{-0.02}^{+0.10}$ & $<160$ & $>10.0$ & $1.61_{-0.03}^{+0.03}$ & $7.07_{-0.24}^{+0.15}$ & $8.2_{-30}^{+24.4}$ & $1.2_{-0.5}^{+0.5}$ & $110_{-40}^{+40}$ & $386 / 381$ & 6 \\
\hline 4 & $0.59_{-0.05}^{+0.04}$ & $210_{-10}^{+10}$ & $5.3_{-1 .}^{+2.8}$ & $1.69_{-0.01}^{+0.02}$ & $6.89_{-0.09}^{+0.12}$ & $6.8_{-16}^{+3.3}$ & $2.1_{-0.5}^{+0.7}$ & $\begin{array}{l}80_{-30}^{+30} \\
30\end{array}$ & $423 / 374$ & 7 \\
\hline 5 & $0.67_{-0.04}^{+0.03}$ & $<8 \times 10^{3}$ & $<0.3$ & $1.63_{-0.10}^{+0.20}$ & $7.17_{-0.47}^{+0.27}$ & $<30$ & $0.7_{-0.5}^{+0.3}$ & $130_{-90}^{+120}$ & $358 / 340$ & 0 \\
\hline 2008 & $0.51_{-0.01}^{+0.01}$ & $60_{-20}^{+20}$ & $<3.9 \times 10^{8}$ & $1.62_{-0.01}^{+0.01}$ & $6.44_{-0.02}^{+0.02}$ & $>210$ & $0.4_{-0.1}^{+0.1}$ & $70_{-10}^{+10}$ & $408 / 384$ & 19 \\
\hline
\end{tabular}

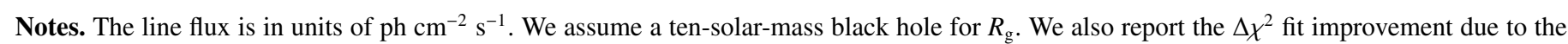
addition of the multicolored disk component.

still statistically unacceptable, especially in the case of OBS1 and OBS2.

The soft X-ray excess could be the signature of the optically thick accretion disk component, which dominates the $\mathrm{X}$-ray emission in the soft state, then smoothly disappears from OBS 1 to OBS5 when the source re-enters in the hard state. To test this hypothesis, we have added a multicolored disk component DISKBB in our fits. The column density is still free to vary independently for the five observations ${ }^{10}$. The best fit parameters

\footnotetext{
${ }^{10}$ Imposing $N_{\mathrm{h}}$ to the same value for the five observations gives a much worse fit with $\Delta \chi^{2}=171$ for four less d.o.f.
} 

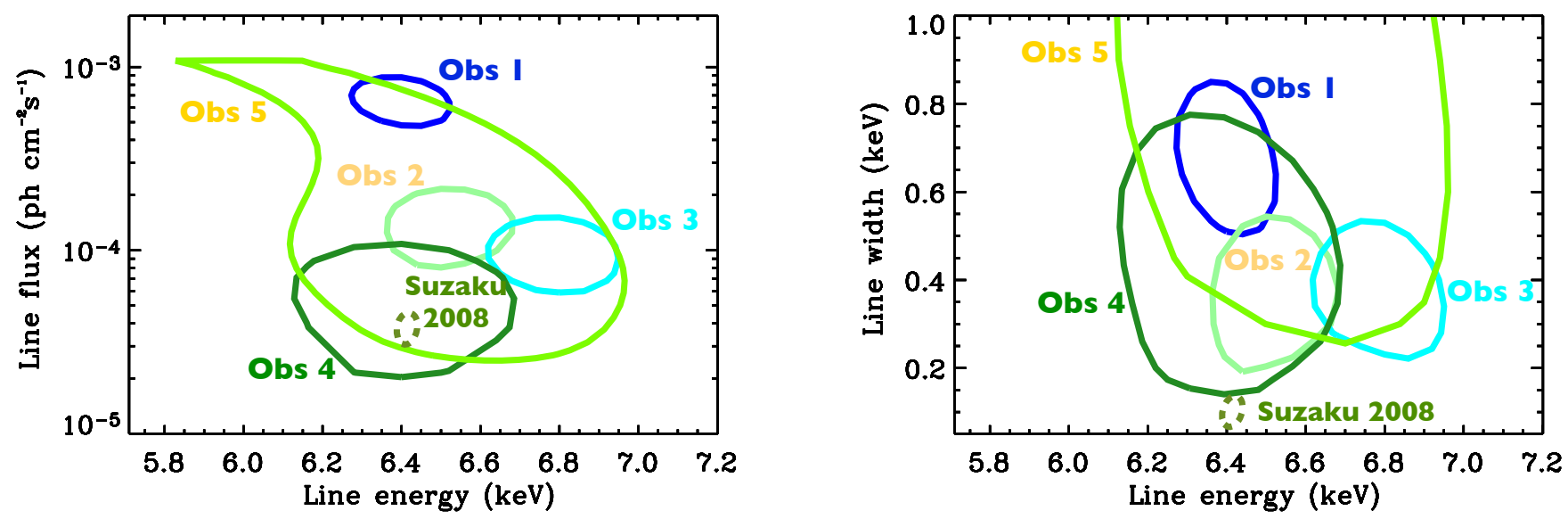

Fig. 4. 90\% contour plots line flux vs. line energy (left) and line width vs. line energy (right) from the best-fits of the five XIS spectra with a POwER LAW + GAUSSIAN model in the 3-10 keV energy range. The dashed contour corresponds to the Suzaku observation of 2008 (Tomsick et al. 2009).

are reported in Table 3. The addition of a multicolored disk component strongly improves the fit of OBS1 and OBS2 with $\Delta \chi^{2}=1332$ and 173 respectively. This component is not statistically required however in the three last observations potentially suggesting the disappearance of the disk component during the state transition.

We have reported the contour plots for the five observations of the DIsквв normalization vs. $R_{\text {in,laor }}$ the best-fit value of the inner disk radius of the LAOR profile in Fig. 7. Assuming an inclination angle of $21 \mathrm{deg}$., a distance of $8 \mathrm{kpc}$, and a black hole mass of 10 solar masses, the DISKBB normalizations can also be converted to an "apparent" disk inner radii $R_{\text {in,app }}$. Taking into account a color temperature correction factor $f_{\text {col }}=1.7$ (Shimura \& Takahara 1995; Kubota et al. 1998; Davis et al. 2006; Done et al. 2012), we deduce the "true" inner radius $R_{\text {in,diskbb }}=f_{\text {col }}^{2} R_{\text {in,app }}$. The values of $R_{\text {in,diskbb have been reported }}$ on the right axis of Fig. $7 \mathrm{a}$.

Both estimates of the disk inner radius (i.e. from DIsKBB or LAOR) roughly agree with each other. However, note a few differences: $R_{\text {in,laor }}$ is consistent between OBS1 and OBS2 while $R_{\text {in,diskbb }}$ of OBS1 is smaller and inconsistent with the OBS2 value. In both cases however, the inner radius is found to be lower than or of the order of $10 R_{\mathrm{g}}$. In OBS3 $R_{\text {in,laor }}$ is constrained to be in between $\sim 5$ and $\sim 40 R_{\mathrm{g}}$ while $R_{\text {in,diskbb }}$ is unconstrained. In OBS5 $R_{\text {in,laor }}$ is upper limited to $\sim 50 R_{\mathrm{g}}$ while $R_{\text {in,diskbb }}$ is unconstrained. We reach however the same conclusions for the evolution of the disk inner radius, $R_{\text {in }}$ which are small in OBS1 and OBS2 $\left(<10 R_{\mathrm{g}}\right)$, but then the contours for OBS3, OBS4 and OBS5 becomes too large to constrain its behavior.

We have checked that these results do not significantly change when using a more physical model (for instance via upscattering of disk photons) for the high energy continuum such as сомPTT in XSPEC. These models indeed differ to a power-law shape especially in the low energy portion of the spectrum where a low energy roll-over is expected. The corresponding contour plots obtained when fitting with сомPTT are reported in Fig. 7b. The main differences with Fig. 7a are larger upper limits on $R_{\text {in,laor }}$ in OBS4 and OBS5.

Note that the fits reported in Table 3 are relatively bad especially for OBS1 and indeed residuals are visible in the soft part $(<2 \mathrm{keV}$, see Fig. 9 and 10) of the spectrum, which indicates that a multicolored disk component alone does not fit the data correctly.

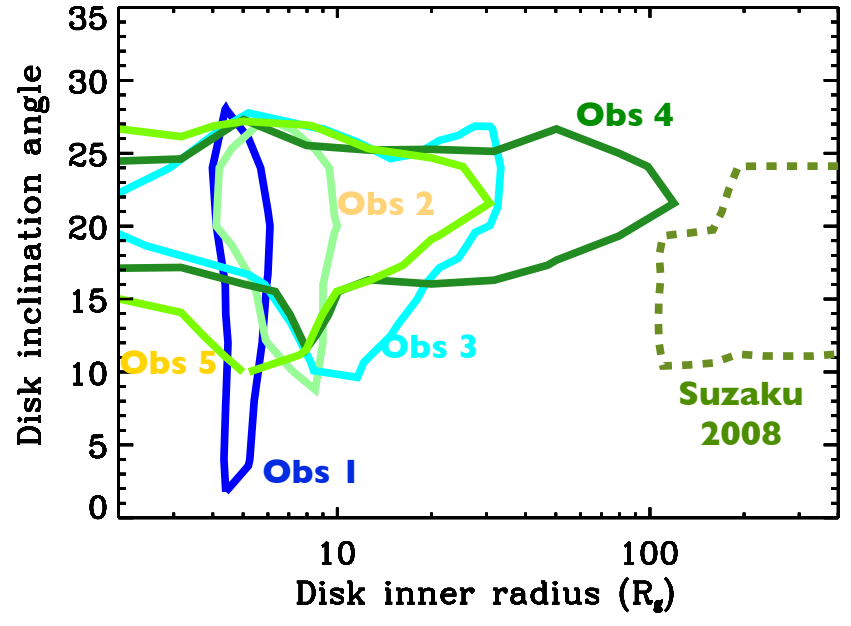

Fig. 5. 90\% contour plots of the disk inclination angle vs. disk inner radius obtained by simultaneously fitting the five XIS spectra with a POWER LAW + LAOR model above $3 \mathrm{keV}$. We impose the same inclination angle between the five models. The dashed contour corresponds to the Suzaku observation of 2008 (Tomsick et al. 2009).

In Fig. 8, we have reported our best-fit results with those obtained by Dinçer et al. (2012). These authors use the RXTE/PCA data during the same outburst decay (from MJD 55559.58 to MJD 55 649.64). They also used a DISKBB + POWER LAW model, and neutral absorption (Due to their limited band pass at low energy, they fixed the hydrogen column density at $\left.5 \times 10^{21} \mathrm{~cm}^{-2}\right)^{11}$, so that our results can be safely compared to theirs. As seen in Fig. 8, both best-fit parameter values agree very well with each other. Compared to RXTE however, the lower limit of the energy band of the XIS Suzaku instrument allows to follow the disappearance of the DISKвB component down to lower flux and lower inner disk temperature.

\subsubsection{Comparison with the 2008 Suzaku observation}

GX 339-4 was observed by Suzaku in 2008 during a long exposure where flux and spectral index values were very close to the one of OBS5 (Tomsick et al. 2009, T09 hereafter). It is worth

\footnotetext{
${ }^{11}$ They also added a smeared edge at a fixed energy of $10 \mathrm{keV}$ to fit the iron $\mathrm{K}$ absorption edge seen around $7.1 \mathrm{keV}$.
} 


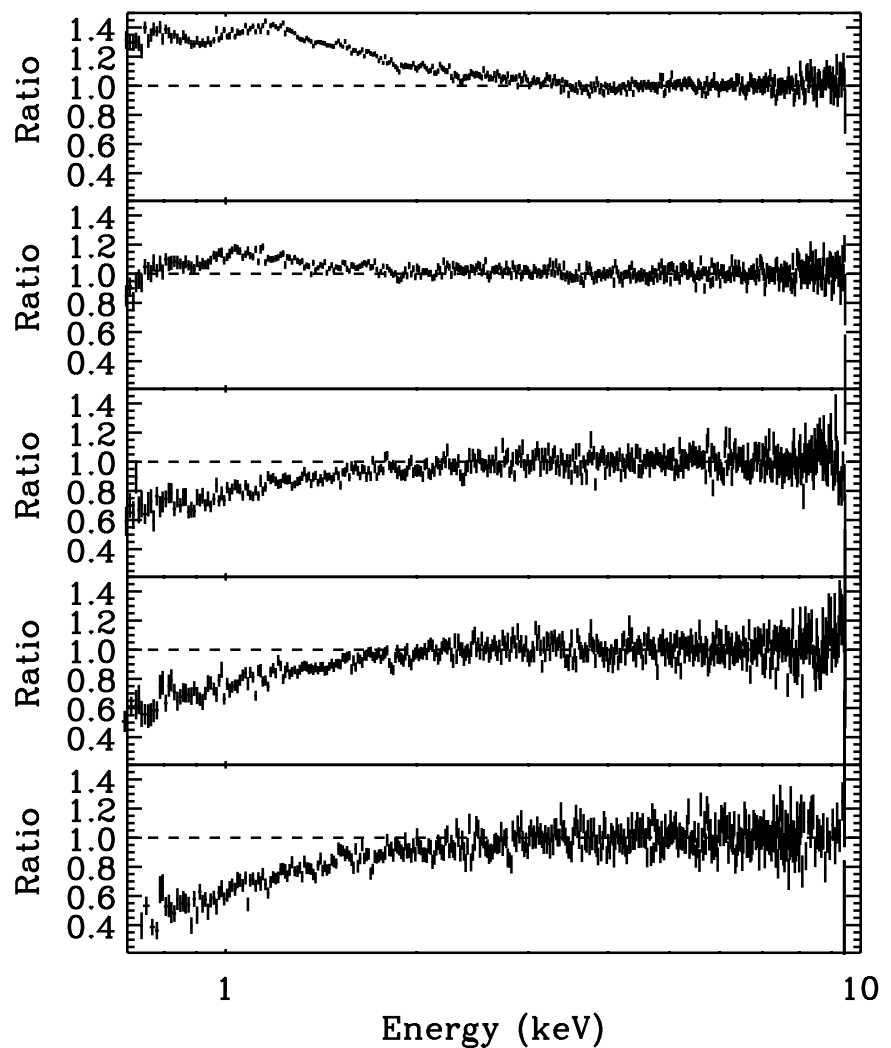

Fig. 6. Data to model ratios of the five XIS0+XIS3 spectra for OBS1 (top) to OBS5 (bottom). The model is a POWER LAW + LAOR fitted above $3 \mathrm{keV}$ and then extrapolated down to $0.7 \mathrm{keV}$. The hydrogen column density is fixed at $6 \times 10^{21} \mathrm{~cm}^{2}$. We use tBNew to model the X-ray absorption (Wilms et al. 2000).

noting, however, that this observation was made 1.6 years after the peak of its 2007 outburst and that the source was in a persistent, but faint, hard state (e.g., Russell et al. 2008; Kong 2008). In comparison, GX 339-4 turned back in the hard state for $\sim 1$ month in 2011, when OBS5 was made, and the source flux was clearly in the decreasing phase of the end of the outburst.

The long exposure (The combined XIS0+XIS3 exposure time is on the order of $210 \mathrm{ks}$ ) results in high statistics compared to our own observations. From the fit of the iron line profile, T09 showed that the data agreed with a truncated accretion disk with $R_{\text {in }}>30 R_{\mathrm{g}}$ at $90 \%$ confidence for a disk inclination of $0 \mathrm{deg}$, and $R_{\text {in }}>70 R_{\mathrm{g}}$ for a disk inclination of $20 \mathrm{deg}$.

We have re-analyzed these data following the procedure detailed in Sect. 2. We have fitted them in the 3-10 keV energy range with the POWER LAW + GAU model first. The corresponding contour plots of the line flux and line width versus line energy are overplotted in Fig. 4 and the best-fit parameter values are reported in Table 2. If the spectral shape and flux agree with OBS5, the line width is clearly inconsistent between the two observations, which indicates intrinsic differences (possibly geometry or ionization state) of the reflecting material.

Then we have fitted these data, which are still in the 3-10 keV energy range, but with the POWER LAW + LAOR model, either separately or simultaneously to our five $S u z a k u$ pointings, imposing, in the latter case, the inclination angle to the same value for each data set. The best-fit results are very similar in both cases and agree with those of T09 (see last row of Table 2). We find an inner radius $>100 R_{\mathrm{g}}$ for 2008 and a best-fit inclination angle of $i=20_{-6}^{+3}$ degrees that are consistent with
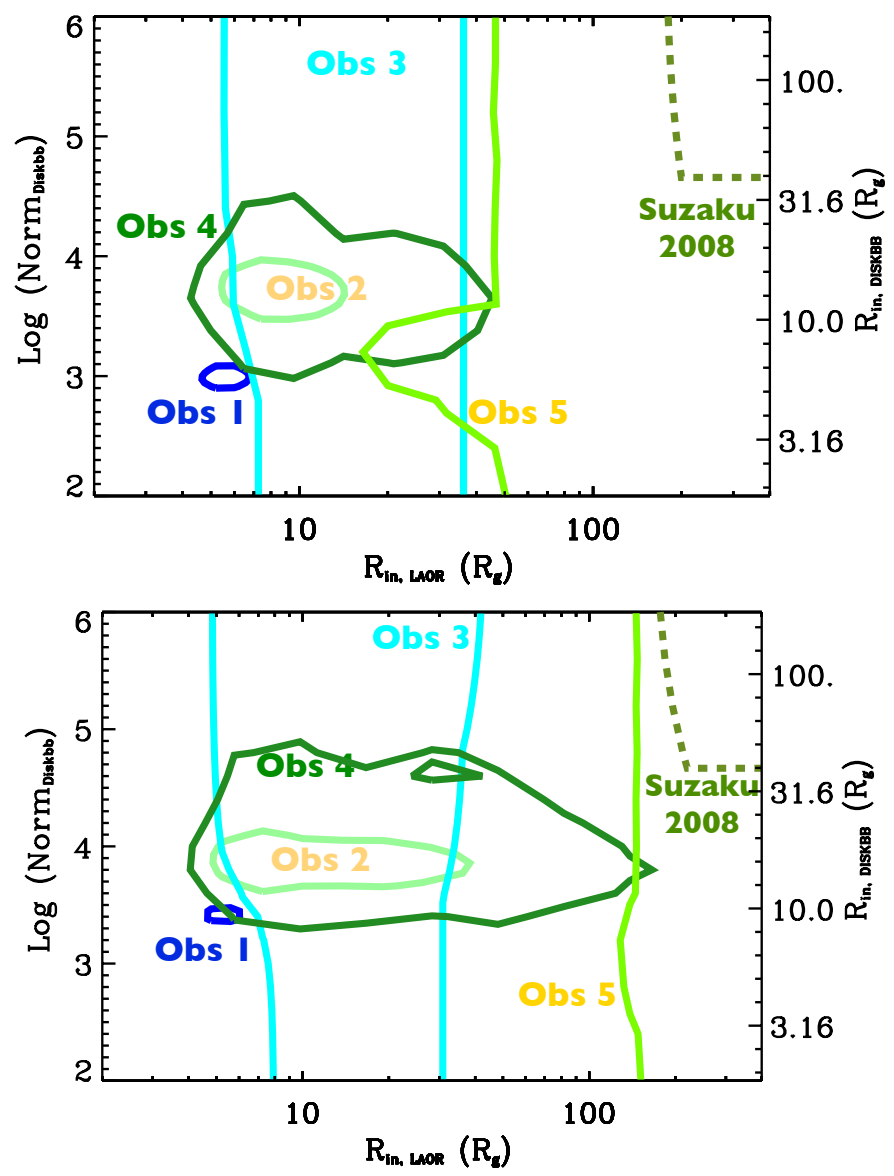

Fig. 7. Contour plots of the disk normalization (left scale) and the corresponding disk inner radius $R_{\text {in,diskbb (right scale) vs. the disk inner ra- }}$ dius $R_{\text {in,laor }}$ deduced from the LAOR profile. The five XIS spectra have been fitted with a DISKBB + LAOR model and a POWER LAW (top) or a comptonization model coMPTT (bottom) for the continuum. We assume a disk inclination of 20 deg., a distance of $8 \mathrm{kpc}$ and a black hole mass of 10 solar masses. For OBS5, the $90 \%$ confidence area is to the left of the light green line. The dashed contour corresponds to the Suzaku observation of 2008 (Tomsick et al. 2009).

our previous value obtained without the use of these data (see Sect. 3.2.1). We have overplotted the $90 \%$ contour plot of the disk inclination angle vs. disk inner radius of the 2008 observation with a dashed line in Fig. 5. While only at a $2 \sigma$ level, the fact that it does not overlap with the $90 \%$ contours obtained for our 2011 observations strongly suggests that the constraints on the disk inner radius that is deduced from the LAOR model are inconsistent between 2008 and 2011.

Following the fitting procedure of the previous sections, we have also fitted the 2008 data set in the $0.7-10 \mathrm{keV}$ range by now adding a DISKBB component to the model. The best-fit results are reported in the last row of Table 3 and the corresponding contour plots of the DISKBB normalization vs. inner disk radius have been overplotted in dashed line in Fig. 7. The constraints on $R_{\text {in }}$ deduced from the DISKBB normalization now give a lower limit of $\sim 30 R_{\mathrm{g}}$ (compared to the lower limit of $\sim 200 R_{\mathrm{g}}$ from the Laor fit of the iron line), which agrees with our (unconstrained) contour plots of OBS3 and OBS5. These constraints on $R_{\text {in }}$ from the DISKBB component have to be taken with caution, given that the disk component is poorly constrained in these observations. 


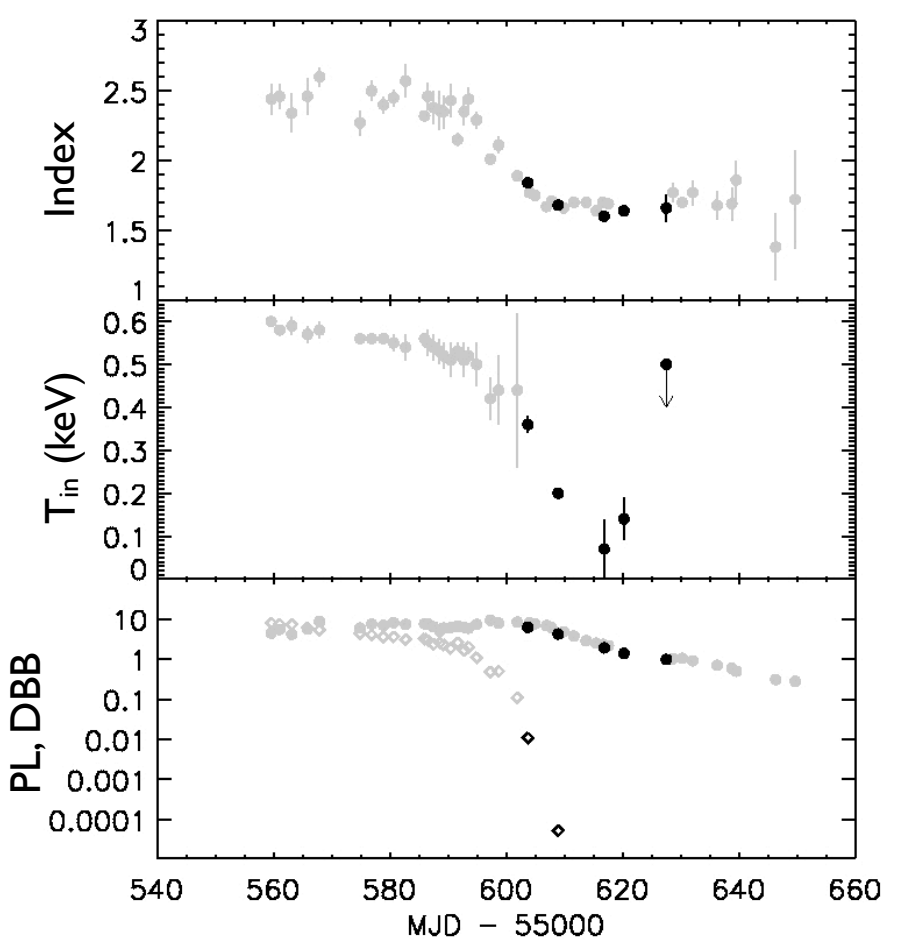

Fig. 8. Evolution of the power law photon index (top), the inner disk temperature $T_{\text {in }}$ (middle) and the power-law and disk flux (bottom, filled and empty circles respectively) in the $3-25 \mathrm{keV}$ energy band in units of $10^{-10} \mathrm{erg} \mathrm{cm}^{-2} \mathrm{~s}^{-1}$. The black symbols are the results of this campaign while the gray ones have been obtained by Dinçer et al. (2012). The model used is TBNEW $\times$ (DISKBB + POWER LAW + GAUSSIAN) in the $0.7-10 \mathrm{keV}$ range.

\subsection{Broadband spectral analysis: ionized reflection}

\subsubsection{Ionized reflection}

From the previous sections, fits of the soft X-ray excess and iron line profiles, which are independent with each other, both suggest an increase of the disk inner radius from OBS1 to OBS2. Its evolution is, however, uncertain for the last three observations. We also confirm the large value of $R_{\text {in }}$ deduced from the iron line fit in the 2008 Suzaku observation. To go a bit further, we use more realistic models for the continuum and the reflection component in this section. We also take advantage of the HXD instrument of Suzaku and include the HXD/PIN (20-70 keV) data in our fits.

In Fig. 9 we have reported the $\chi^{2}$ contribution when we extend the best-fit model TBNEW $\times$ (DISKBB + POWER LAW + LAOR) of the XIS data (the best-fit parameters are reported in Table 3) above $10 \mathrm{keV}$ in the HXD/PIN energy range. A clear excess between 20 and $40 \mathrm{keV}$ is visible especially in OBS1 and OBS2 and suggests the presence of a reflection bump.

To provide a more physical description of the reflection component that gives birth to the iron line, we use the combination of the REFLIONX code of Ross \& Fabian (2005), which is convolved with the relativistic kernel KDBLUR (Laor 1991). In agreement with the results of Sect. 3.2.1, we fix the outer disk radius $R_{\text {out }}$ to $400 R_{\mathrm{g}}$ in KDBLuR, the index of the radial power law dependence of the emissivity to 3 and the disk inclination angle to 20 degrees.

In REFLIONX, the illumination has a power law shape. We first fit the different observations with a power-law for the primary continuum, by fixing the photon index in REFLIONX to that

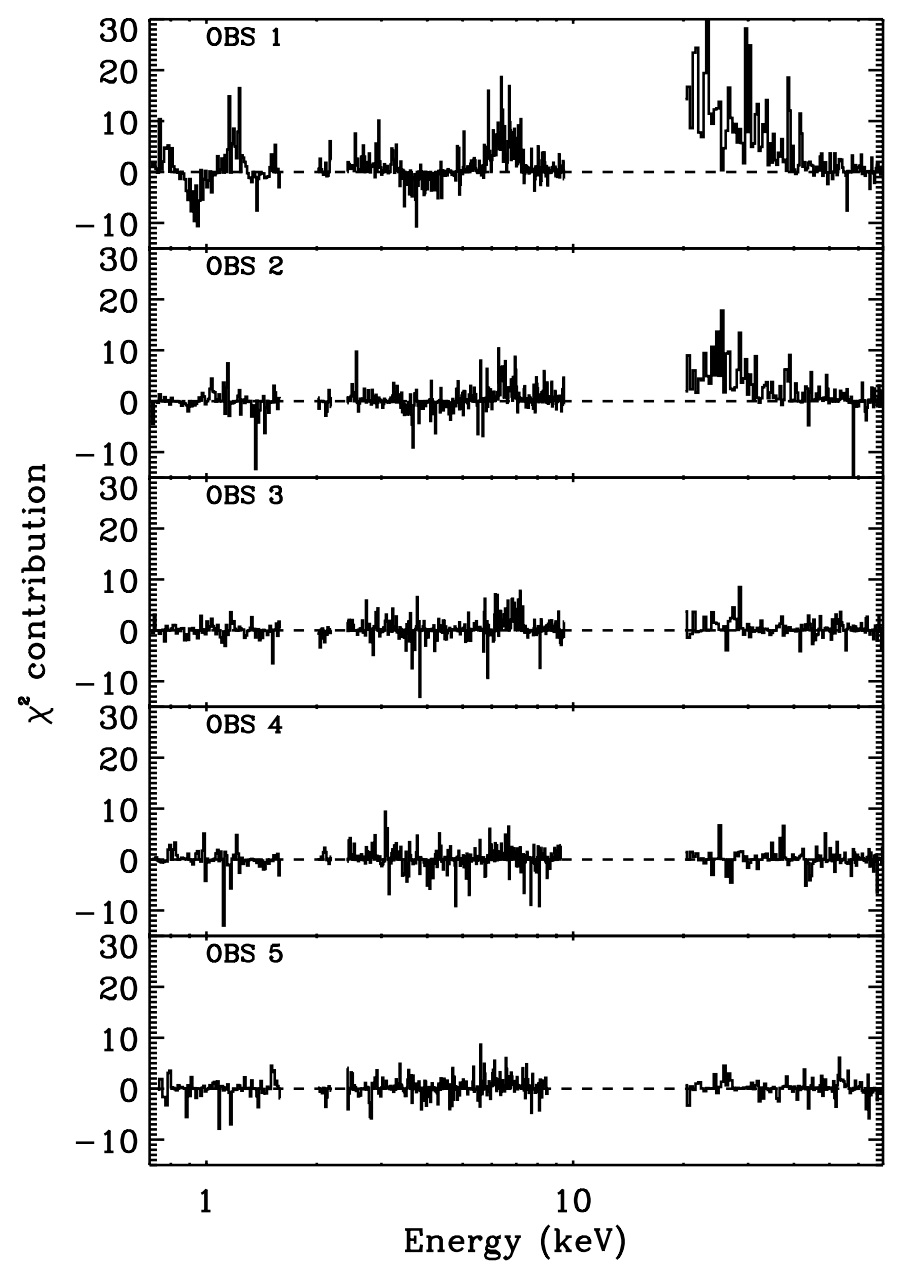

Fig. 9. $\chi^{2}$ contribution in the $0.7-70 \mathrm{keV}$ range when extending the best fit model TBNEW $\times$ (DISKBB+ POWER LAW + LAOR) (see Table 3$)$ of the XIS data above $10 \mathrm{keV}$ in the PIN energy range. The LAOR component has been suppressed in these plots. Strong residuals are visible near $6.4 \mathrm{keV}$ and above $10 \mathrm{keV}$ especially for OBS1 and OBS2.

of the power law continuum. However, as already discussed in Sect. 3.2.2, comptonization of the soft disk photons in a hot corona is widely accepted as the mechanism at the origin of the X-ray continuum of X-ray binaries. Thus, instead of a simple power law, we also use the Comptonization model EQPAIR (Coppi 1999) even if it is not perfectly consistent with the use of REFLIONX. In this case, we fix the photon index in REFLIONX to the precedent values obtained when fitting with the power law continuum $^{12}$. We see that our results are qualitatively similar between the two models the advantage of EQPAIR is that its main parameters (i.e. the ratio between the compactness of seed photons $l_{\mathrm{s}}$, and hot electrons $l_{\mathrm{h}}$, the corona optical depth $\tau$ and the temperature of the soft disk photons $T_{\mathrm{bb}}$ ) have a direct physical meaning.

The best-fit parameters obtained with these models are reported in Table 4. Note that the fits are always better with EQPAIR than with a power law. The lack of a low energy roll-over in this latter case could explain the larger residuals observed in the soft band $(<2 \mathrm{keV})$. The best-fit parameters are however qualitatively similar between the two models, the main difference being the

12 The slope of EQPAIR can be estimated from the $l_{\mathrm{h}} / l_{\mathrm{s}}$ ratio (e.g., Malzac et al. 2001) and appears to be very close to the photon index of the power law fits. 
P.-O. Petrucci et al.: The return to the hard state of GX 339-4 as seen by Suzaku
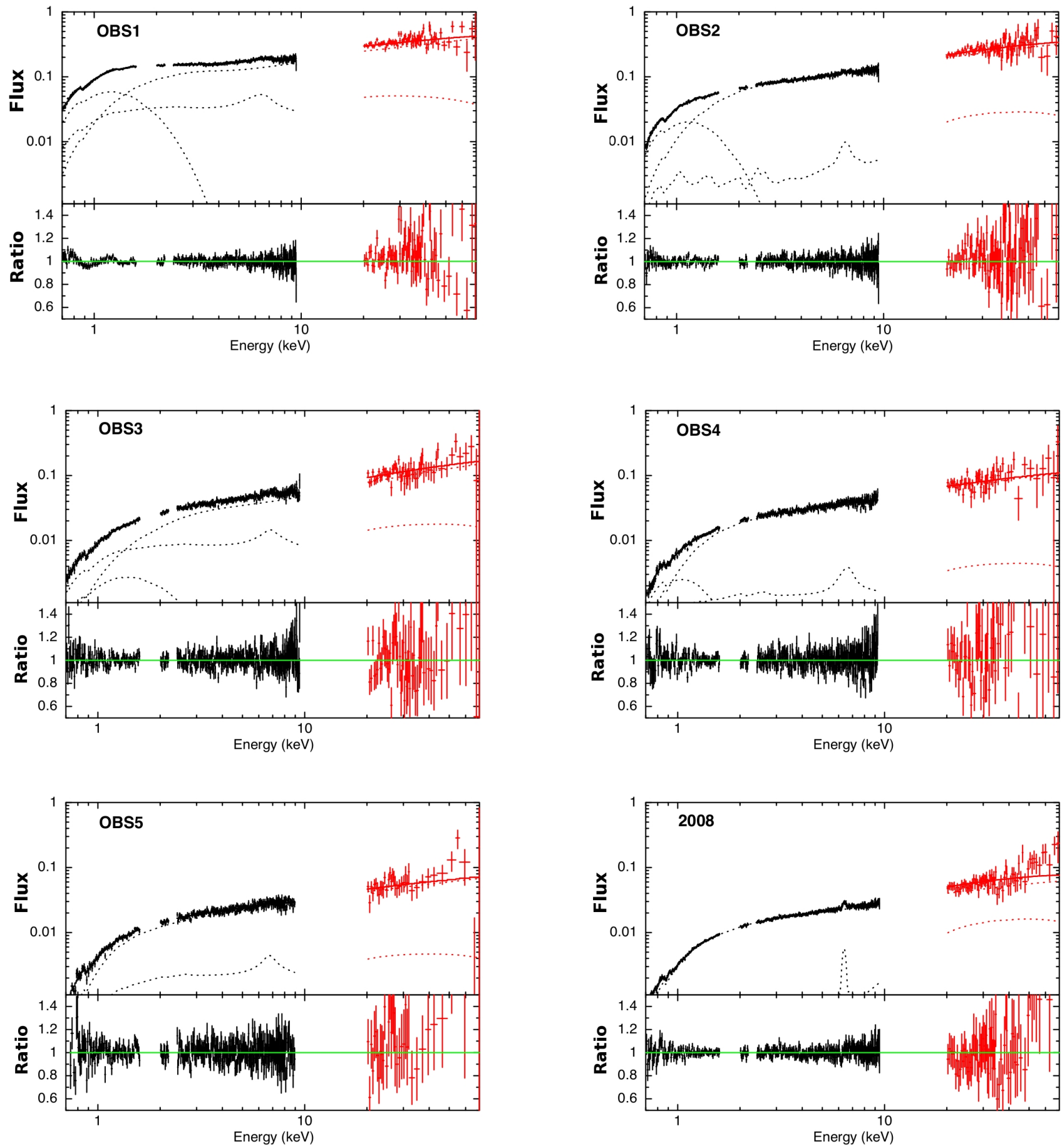

Fig. 10. Unfolded best-fit model of the five Suzaku observations (XIS: black crosses; HXD/PIN: red crosses) and corresponding data/model ratios using EQPPAIR for the continuum, a blurred reflection KDBLUR $\otimes$ REFLIONX for the reflection component and a multicolored disk DISKBB for the soft X-rays.

reflection component in OBS1 which has a lower flux and lower ionization with the use of a power law continuum. However, $R_{\text {in }}$ is still well constrained to a small value $\sim 15 R_{\mathrm{g}}$ in this observation. We note also that the ionization parameter is not larger in OBS3 compared to the other observations and that does not support the presence of a more ionized iron line as suggested by the fits between 3 and $10 \mathrm{keV}$ with a simple Gaussian (see Sect. 3.2.1).

Since both models (with a power law or EQPAIR for the continuum) give similar parameter constraints and since the fits with EQPAIR always give a better $\chi^{2}$, we only discuss the results obtained with this model from now on. We find good fits in all cases but some features in OBS1 are still present in the soft energy range $(<1 \mathrm{keV}$, see next section). The unfolded spectra and the data/model ratios of each observation are reported in Fig. 10. To make a direct comparison to the DISKBB + POWER LAW + GAUSsian fits below $10 \mathrm{keV}$ as discussed in Sect. 3.2.2 (and reported in Table 3), we have computed the corresponding $\chi^{2} /$ d.o.f. of these new fits but limited to the $0.7-10 \mathrm{keV}$ energy range. We find the following values: 530/382, 401/382, 392/379, 417/372 
Table 4. Results of the fits of the XIS and HXD data with a POWER LAW (top part) or EQPAIR (bottom part) for the continuum and the ionized reflection component REFLIONX.

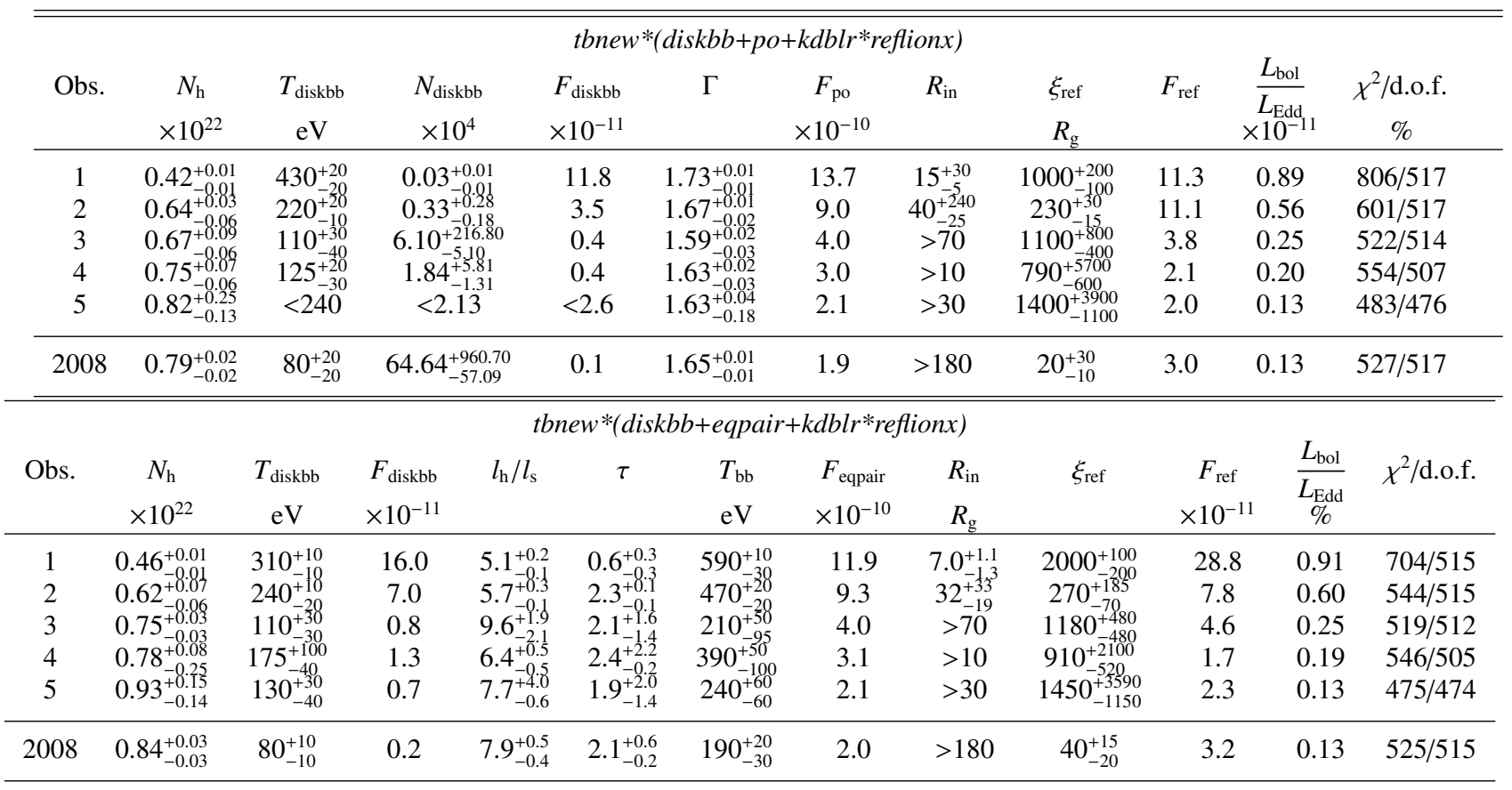

Notes. In the case of the POWER LAW fit, the photon index used in REFLIONX is fixed to the one of the power law continuum. In the case of EQPAIR, we froze the photon index of REFLIONx to the one obtained in the power law fit. The inclination was fixed to 20 deg. We use the kernel from the LAOR line profile to account for the gravitational effects close to the black hole (model KDBLUR) of xSPEC. All errors have a 90 per cent confidence for one parameter. The fluxes are in units of $\mathrm{erg} \mathrm{cm}^{-2} \mathrm{~s}^{-1}$ and are computed in the $0.7-70 \mathrm{keV}$ energy range. The bolometric luminosity $L_{\mathrm{bol}}$ is assumed to be the sum of the luminosities of the three spectral component DISKBB, EQPAIR and REFLIONX. We adopt an Eddington luminosity of $L_{\mathrm{Edd}} \simeq 1.3 \times 10^{39} \mathrm{erg} \mathrm{s}^{-1}$ (i.e. we assume a 10-solar-mass black hole) and a distance to GX $339-4$ of $8 \mathrm{kpc}$.

and 355/341 for the five (from OBS1 to OBS5) observations respectively. The improvement of the fits in the $0.7-10 \mathrm{keV}$ energy range with the blurred ionized reflection model are really significant for OBS1 and OBS2 with $\Delta \chi^{2}=49$ and 27 for two less degrees of freedom. No significant improvement is obtained for the other observations, potentially because of their lower statistics.

The hard to soft compactnesses ratio $l_{\mathrm{h}} / l_{\mathrm{s}}$ increases smoothly along all the monitoring which agrees with the observed spectral hardening (see Table 2). It is on the order of 5-8, which suggests a photon starved geometry for the hot corona. On the other hand, the hot corona optical depth, $\tau$, increases significantly between OBS1 and OBS2 by a factor $\sim 4$ and then stays roughly constant on the order of $\sim 2-3$. Concerning the ionization parameter, apart from OBS2, it is relatively high (with admittedly large error bars) on the order of $\xi_{\text {ref }} \sim 1000$, which suggests a ionized reflecting medium.

The 0.7-70 keV fluxes of the different spectral components (respectively $F_{\text {diskbb }}, F_{\text {eqpair }}$ and $F_{\text {ref }}$ ) are also reported in Table 4 . They show a clear decrease along the monitoring, which agrees with when the source returns back to its quiescent state. However, the relative ratios $F_{\text {diskbb }} / F_{\text {eqpair }}$ and $F_{\text {ref }} / F_{\text {eqpair }}$ are not constant, as we expect if all the spectral components fade in the same way. These ratios decrease from $\sim 14$ to $1 \%$ and $\sim 25$ to $10 \%$ respectively.

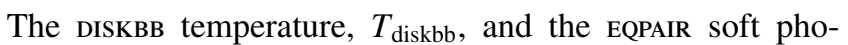
ton temperature, $T_{\mathrm{bb}}$, also show a clear decrease from OBS1 to OBS5. Interestingly, while the two temperatures were let free to vary during the fitting procedure, $T_{\text {diskbb }}$ is about half of $T_{\mathrm{bb}}$ (see Fig. 11a). This correlation may have a physical origin. Indeed, the soft temperature $T_{\mathrm{bb}}$ in EQPAIR corresponds to the disk temperature actually "seen" by the hot corona. On the other hand, $T_{\text {diskbb }}$ is the effective disk temperature. The ratio between the two, the so-called spectral hardening factor, is generally estimated to be on the order of 2-3 (e.g., Shimura \& Takahara 1995; Sobczak et al. 1999; Merloni et al. 2000; Davis et al. 2006), which agrees with what we find.

For comparison we have also fitted the 2008 Suzaku observation with the same model. The best-fit parameters are reported in the last row of Table 4. Apart from the reflection ionization parameter, which is on the order of $\sim 40$ i.e. well below our best-fit values that are observed in 2011 and a larger disk inner radius, the other parameters agree well with the spectral evolution from OBS1 to OBS5. More precisely, even if there is a 3-year gap between them and a poor statistics in 2011 , the 2008 observation seems to be very similar to OBS5 but with a different accretion disk state.

We have checked if the uncertainties on $R_{\text {in }}$ in OBS5 could be due to the lower statistics compared to 2008 by simulating a set of data from the best-fit model obtained for 2008 but with a combined XIS0+XIS3 exposure time of $40 \mathrm{ks}$ (i.e. on the order of the exposure time of OBS5) instead of $210 \mathrm{ks}$. A fit of this simulated spectrum gives $R_{\text {in }}>130 R_{\mathrm{g}}$ and $\xi_{\text {ref }}=70_{-20}^{+60}$. If the OBS5 spectrum and line shape were exactly the same as in 2008, we should have obtained values of $R_{\text {in }}$ and $\xi_{\text {ref }}$ which agree with those observed in 2008, even if the statistics of OBS5 are low. This is in contradiction with our results and this suggests that the accretion disk is in an intrinsically different state between 2008 and 2011. 

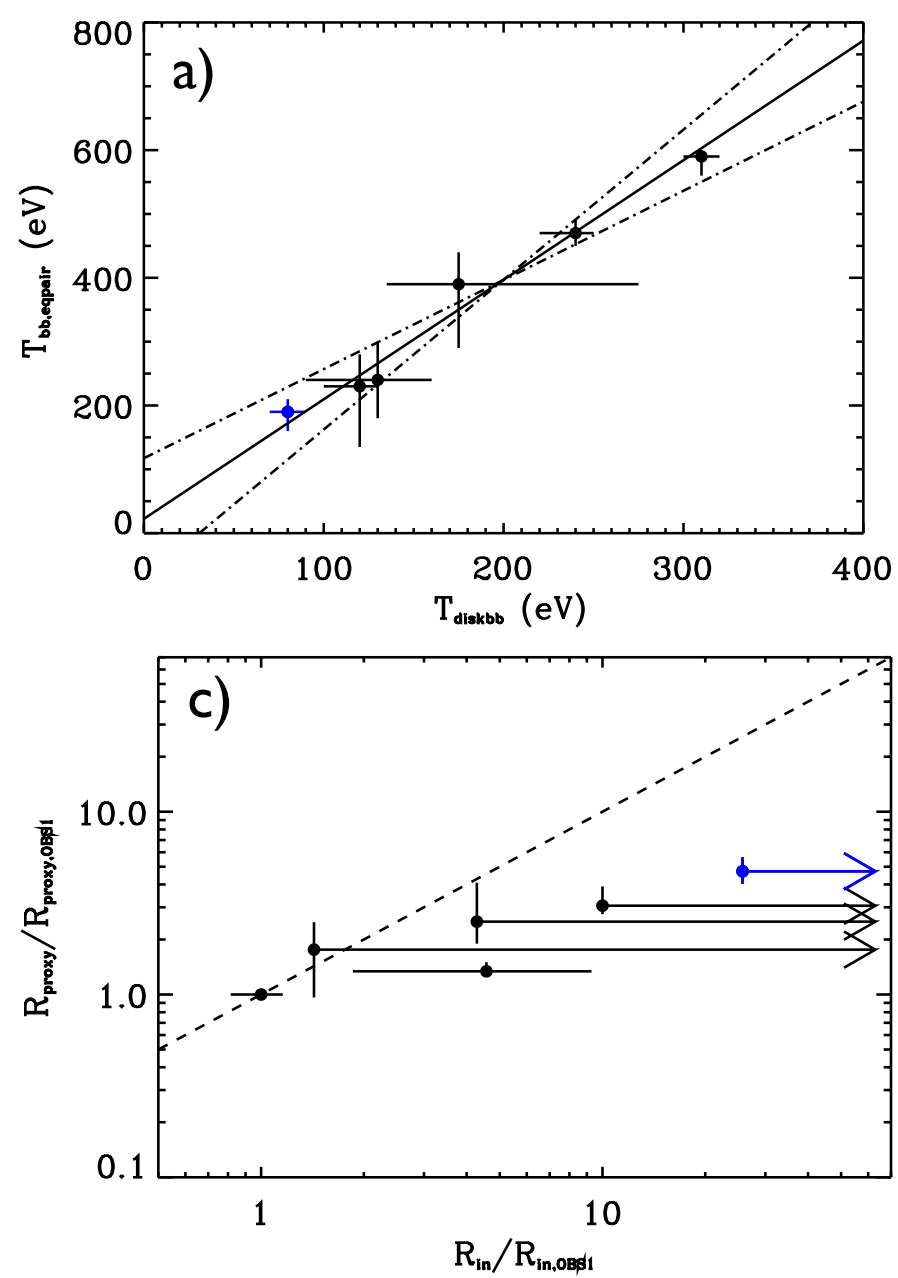

\subsubsection{Ionized absorption}

As said previously, the best fit of OBS1 is not very satisfactory and features are visible, especially in the soft X-ray range, in the data to model ratio reported in Fig. 10. The presence of absorption lines in the soft X-ray spectrum of GX 339-4 have already been reported in the literature when the source was in an intermediate state transiting to the low/hard state (e.g., M04, Juett et al. 2006). If most of these lines may be produced by the interstellar medium (Juett et al. 2006), a sizable contribution of a few of them (e.g., the $\mathrm{Ne}$ Ix line at $0.922 \mathrm{keV}$ ) could be due to a local contribution from an intrinsic AGN-like warm-absorber which is perhaps produced by a disk wind.

We have tested the presence of this absorber by adding an ionized absorber component (model ABSORI of XSPEC) in our fits. The fit of OBS1 improves significantly $\left(\Delta \chi^{2}=102\right.$ !) thanks to the addition of this spectral component. The corresponding parameters of the absorber are reported in Table 5 that is an hydrogen column density $N_{\mathrm{h}, \mathrm{abs}} \sim 10^{22} \mathrm{~cm}^{-2}$ and an ionization parameter $\xi_{\text {abs }} \sim 300$. The other model parameters do not significantly change compared to Table 4 . We add the same ABsori component to the other observations (including 2008). We do not find any significant improvement. The best fit are also reported in Table 5. The decrease of the signal-to-noise ratio may, however, limit the correct detection of absorbing material ${ }^{13}$.

\footnotetext{
${ }^{13}$ Despite the difference in the soft band due to the lack of a low energy roll-over when fitting the continuum with a power law, we obtain consistent values of the absorber parameters when we use this model instead of EQPAIR.
}

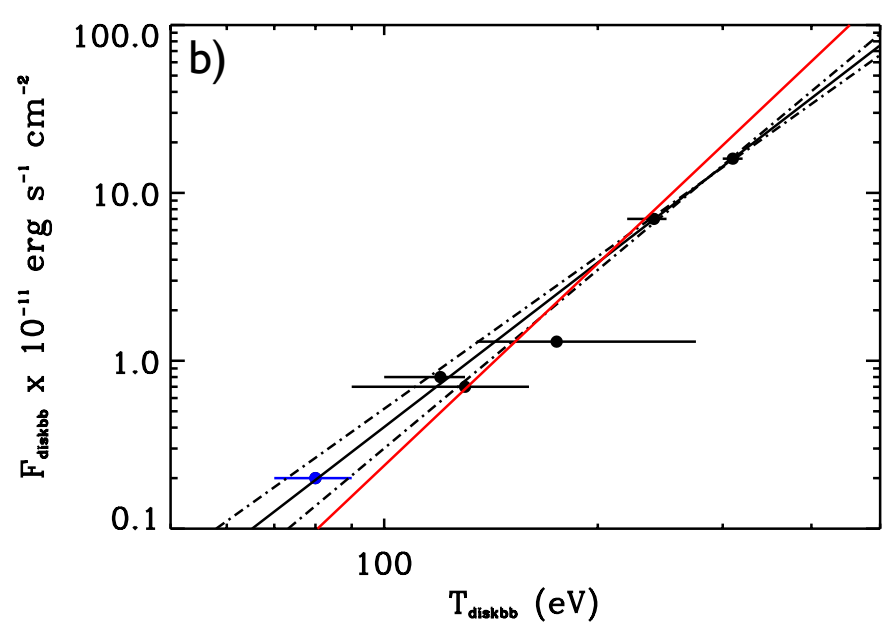

Fig. 11. a) Best-fit DISKBB temperature vs EQPAIR soft photon temperature. The solid line is the linear best fit. It has a slope of 1.9. The dot-dashed lines correspond to the $1 \sigma$ error on the slope i.e. $1.9 \pm 0.5$. b) Flux of the рікквв component versus Disквв temperature. The solid black line corresponds to the $\log \log$ linear best fit $F_{\text {diskbb }} \propto T_{\text {diskbb }}^{3.3}$ and the dot-dashed lines to the $1 \sigma$. The solid red line corresponds to the best fit assuming a $T_{\text {diskbb }}^{4}$ law. c) Plot of $R_{\text {proxy }}$ (see Sect. 4) versus the disk inner radius $R_{\text {in }}$ deduced from our fits (reported in Table 4) and which assumes the X-ray luminosity $\propto \dot{M}^{\alpha}$ with $\alpha=2.5$ (the results are very similar for $\alpha=2$ or 3 ). The dashed line corresponds to $R_{\text {proxy }}=R_{\text {in. }}$. The blue points in each figure correspond to the 2008 Suzaku observation.

Table 5. Best fit parameters of the ionized absorber component.

\begin{tabular}{lcccc}
\hline \hline \multicolumn{5}{c}{ Ionized absorber } \\
Obs. & $N_{\mathrm{h}}$ & $\xi_{\text {abs }}$ & $\Delta \chi^{2}$ & $\chi^{2} /$ d.o.f. \\
& $\times 10^{22}$ & & & \\
\hline 1 & $1.0_{-0.2}^{+0.2}$ & $320_{-60}^{+90}$ & 107 & $597 / 513$ \\
2 & $0.4_{-0.3}^{+0.1}$ & $180_{-130}^{+230}$ & 3 & $541 / 513$ \\
3 & $<4.0$ & $>90$ & -1 & $520 / 510$ \\
4 & $<2.4$ & {$[0-5000]$} & 1 & $551 / 503$ \\
5 & $7.4_{-3.7}^{+3.2}$ & $>4900$ & 3 & $472 / 472$ \\
\hline \multirow{2}{*}{2008} & $<0.25$ & {$[0-5000]$} & 0 & $524 / 513$ \\
\hline
\end{tabular}

Notes. The $\Delta \chi^{2}$ is the $\chi^{2}$ variation compared to the best fits reported in Table 4.

Then, we can roughly estimate the maximal distance $d$ between the absorbing material and the X-ray source by assuming that $d$ is necessarily larger than the radial extension $\Delta r$ of the absorber. Since $N_{\mathrm{h}, \mathrm{abs}}=n \Delta r$ (n being the density of the warm absorber) and $\xi_{\mathrm{abs}}=\frac{L_{\mathrm{bol}}}{d^{2} n}$ then $d>\Delta r$ implies $d<\frac{L_{\mathrm{bol}}}{\xi_{\mathrm{abs}} N_{\mathrm{h}, \mathrm{abs}}}$. Using the best fit parameter values obtained for OBS1 i.e. $L_{\mathrm{bol}} \simeq$ $10^{37} \mathrm{erg} \mathrm{s}^{-1}, \xi_{\mathrm{abs}} \simeq 300 \mathrm{erg} \mathrm{cm} \mathrm{s}^{-1}$ and $N_{\mathrm{h}, \mathrm{abs}} \simeq 10^{22} \mathrm{~cm}^{-2}$ we find that the absorber should be at a maximum distance of $\sim 10^{6} R_{\mathrm{g}}$ (assuming a 10 black hole solar masses) from the central X-ray source. This is on the order of the binary separation in GX 339-4 as estimated by Zdziarski et al. (2004), thus it agrees 
that the absorber may be produced in the inner parts of the binary system.

To have a qualitative idea of the ions potentially responsible for the observed absorption features in OBS1, instead of ABSORI we simply add Gaussian absorption lines, with widths fixed to $0 \mathrm{eV}$ (a more detailed work on these absorption line is dedicated to a future work). We focus only on OBS1 since the other observations do not apparently need the addition of absorption components. As previously said, a few absorption lines were already observed in GX 339-4 in past Chandra observations (M04), with the most intense one being Ne Ix at $0.922 \mathrm{keV}$. We thus add an absorption Gaussian line with energy in the range $0.9-0.95 \mathrm{keV}$. The fit improves strongly with $\Delta \chi^{2}=61$ for 3 less degrees of freedom. The Gaussian best fit energy $E_{\text {abs }}=0.92 \pm 0.02$ is consistent with $\mathrm{Ne} \mathrm{IX}^{14}$. The line has an equivalent width $(\mathrm{EW})$ of $\sim 5.7 \mathrm{eV}$ which corresponds to a Neon column density in between $5 \times 10^{16}$ and $10^{17} \mathrm{~cm}^{-2}$ (see M04 for the computation of the Neon column density). This agrees completely with the estimates measured by M04 and Juett et al. (2006) and this is still higher than the expected value from the ISM, which suggests an origin in the local environment of the X-ray binary. Luo \& Fang (2014) reach the same conclusion for GX 339-4 and for a sample of 11 other $\mathrm{X}$-ray binaries. We have tested that the addition of this Gaussian absorption line is not needed in the other observations.

We have also tested the presence of (weak) blue-shifted ionized absorption lines from Fe XXv and Fe XxvI since they have been observed in a few microquasars and interpreted as the signature of fast ionized outflows. Their expected EW are generally on the order of 10-20 eV (e.g., Ponti et al. 2012). We do not detect such components in our data and find only upper limit for their EW of 5-10 eV.

\section{Hints of disk recession}

To be correct, when we talk about disk recession, we are talking about the recession of the optically thick part of the accretion flow. It is possible that the accretion disk extends down to the innermost stable circular orbit but that, for different reasons (e.g., most of its accretion power is advected or ejected), it does not radiate any more.

While we do not find clear indications of disk recession during our monitoring, a few arguments are consistent with this interpretation. Contrary to fits below $10 \mathrm{keV}$, the fits of the broadband spectra with blurred ionized reflection give good constrains on $R_{\text {in }}$ for OBS1 and OBS2, which are on the order of $10 R_{\mathrm{g}}$ and $\sim 30 R_{\mathrm{g}}$ respectively, but it also puts lower limits for OBS3 $\left(>70 R_{\mathrm{g}}\right)$, OBS4 $\left(>10 R_{\mathrm{g}}\right)$ and OBS5 $\left(>30 R_{\mathrm{g}}\right)$. For the 2008 data set, our fit gives a lower limit $R_{\text {in }}>180 R_{\mathrm{g}}$, which agrees with T09 and clearly suggests a disk recession (see Fabian et al. 2014 for the limitations of the use of the X-ray reflection to estimates the disk inner radius). This recession was apparently stronger in 2008 which could be due to the fact that the source was in a persistently faint hard state for months. This could have given enough time for the inner accretion flow to evolve into the observed configuration.

Our estimates of $l_{\mathrm{h}} / l_{\mathrm{s}}$ from our fits also agree with the work of Sobolewska et al. (2011). Note however that we are not using exactly the same model as these authors. No reflection component was taken into account in their work expect for an iron line. We do not expect, however, that this would have any strong effect on our estimate of $l_{\mathrm{h}} / l_{\mathrm{s}}$ compared to their methods given the

\footnotetext{
${ }^{14}$ Note that this absorption line energy is well below the Si edge which is known to contaminate the XIS response around $1.8 \mathrm{keV}$.
}

low flux we found in the reflection component (i.e. a factor 10 below the continuum flux, see Table 4). Thus we believe that our results can be safely compared to theirs. These authors studied the changes of the $l_{\mathrm{h}} / l_{\mathrm{s}}$ ratio with the bolometric luminosity $L_{\mathrm{bol}}$ in the hard states of GX 339-4 and GRO J1655-40. At luminosities on the order of $\sim 0.1-1 \%$, i.e. the range of luminosities of the present Suzaku observations, Sobolewska et al. (2011) confirmed a transition in behavior of $l_{\mathrm{h}} / l_{\mathrm{s}}$ with $L_{\mathrm{bol}}$. At luminosities lower than $\sim 0.1-1 \%$, both parameters correlate while they anticorrelate at larger $L_{\mathrm{bol}}$. According to these authors, this behavior is consistent with a scenario where seed photons change from cyclo-synchrotron at the lowest luminosities to those from a (truncated) disk at higher luminosities. Our observed increase of $l_{\mathrm{h}} / l_{\mathrm{s}}$ from OBS1 to OBS5, with a decrease of $L_{\mathrm{bol}}$, suggests then that the emission of the accretion disk still dominates the cooling process of the hot corona.

However, the changes of $l_{\mathrm{h}} / l_{\mathrm{s}}$ implies a variation of the diskcorona geometry. The increase of $l_{\mathrm{h}} / l_{\mathrm{s}}$ from OBS1 to OBS5 indicates a faster decrease of the soft photon flux compared to the corona heating power. This situation is naturally expected if $R_{\text {in }}$ increases from OBS1 to OBS5. Note that the increase of $R_{\text {in }}$ would also imply a diminution of the reflecting area and consequently a faster decrease of the reflecting component compared to primary one. This also agrees with the decrease of $F_{\text {ref }} / F_{\text {eqpair }}$ as discussed in Sect. 3.3.1.

We have reported the flux $F_{\text {diskbb }}$ of the DISKBB component versus the DISKBB temperature $T_{\text {diskbb }}$ in Fig. $11 \mathrm{~b}$. The log-log linear best fit (solid black line in Fig. 11b) gives $F_{\text {diskbb }} \propto T_{\text {diskbb }}^{3.3 \pm 0.2}$ which is a bit smoother than the Stefan-Boltzmann law $\propto T^{4}$ which is expected in the case of an optically thick accretion disk with fixed inner radius. This discrepancy could be explained by a variation of the hardening factor with the disk temperature (e.g., Salvesen et al. 2013). However, we can again interpret this result in term of a variation of the disk inner radius. Indeed, we expect, in an standard accretion disk of inner radius $R_{\text {in }}$, inner temperature $T_{\text {diskbb }}$ and accretion rate $\dot{M}$ :

$T_{\text {diskbb }}^{4} R_{\text {in }}^{3} \propto \dot{M}$.

On the other hand, X-ray binaries are known to be in a radiatively inefficient state where the $\mathrm{X}$-ray luminosity is proportional to $\dot{M}^{\alpha}$ with $\alpha \sim 2-3$ (e.g., Coriat et al. 2011 an references therein) at the end of the outbursts. Then $F_{\text {eqpair }}^{1 / \alpha}$ can be used as a proxy for the accretion rate of our system. In consequence, the ratio $R_{\text {proxy }}=\left[F_{\text {eqpair }}^{1 / \alpha} / T_{\text {diskbb }}^{4}\right]^{\frac{1}{3}}$ should be a proxy of the disk inner radius $R_{\text {in }}$. We have plotted $R_{\text {proxy }}$ normalized to its value in OBS1 (and assuming $\alpha=2.5$ ) in Fig. 11c versus the bestfit inner radius values $R_{\text {in }}$ that are obtained from our broadband fits. While the values of $R_{\text {proxy }}$ for the last four observations are always larger than the one computed for OBS1, which then supports the scenario of the recession of the optically thick part of the accretion disk, they are smaller than $R_{\text {in }}$ in most cases. This discrepancy may be due to the bad estimate of the disk inner temperature $T_{\text {diskbb }}$ from our fits due to the limited energy range of Suzaku in the soft X.

\section{Disk-wind to disk-jet transitions?}

In the recent context of outflow/wind signature detection in black hole X-ray binaries (e.g., Miller et al. 2006c; Ponti et al. 2012; Díaz Trigo et al. 2012) and their potential link to the jet evolution during an outburst (Miller et al. 2006d; Neilsen \& Lee 2009), the absorption features significantly detected in OBS1 (and only in OBS1) is an interesting result. Let us recall that the re-ignition 
of the radio emission was detected just before the beginning of our campaign, which peaks between OBS1 and OBS2, and is interpreted as the beginning of the jet-structure re-building while the source turned back to the hard state (e.g., C13). By analogy with the observation of the evolution between a jet-dominated to a wind-dominated accretion flow during a hard-to-soft transition in GRS 1915+105 (Neilsen \& Lee 2009), our results could correspond to the reverse situation, that is the evolution from a wind-dominated to a jet-dominated accretion flow during a softto-hard transition.

The absorption features in OBS1 agree with the presence of Neon absorption lines, especially from Ne Ix and its column density suggests that part of this line could be produced locally. Interestingly, this line was detected in a Chandra observation of GX 339-4 when the source was in an intermediate state transiting to the low/hard state while OBS1 was observed just before the complete state transition back to the hard state. The disappearance of the Ne Ix in the other observations of the monitoring could suggest that it is linked to a disk wind that is only present before the transition to the hard state. The absence of detection of Fe xxv and Fe xxvI however seems to indicate that this wind, if really present in OBS1, is not highly ionized.

\section{Concluding remarks}

Our Suzaku monitoring of GX 339-4 at the end of its 2010-2011 outburst caught the source during its soft-to-hard state transition. Simultaneous radio-OIR observations showed the recovery of the radio emission and were interpreted as a signature of the re-ignition of the compact jets (C13). The onset of the radio emission occured between OBS1 and OBS2 while the reflare observed in OIR reached its maximum close to OBS3 and OBS4.

The Suzaku observations show a global fading of the X-ray flux during the monitoring. For the spectral fits, we first use phenomenological models with a simple power law for the continuum. The addition of an iron line is statistically needed in all the observations. Fits with a LAOR profile are statistically equivalent to fits with a Gaussian. Simultaneous fits of the 5 observations with a LAOR profile give a disk inclination angle of $\sim 20 \mathrm{deg}$. The constraints on the inner radius agree with $R_{\text {in }}<10 R_{\mathrm{g}}$ in OBS1 and OBS2 at a $90 \%$ confidence level. Due to the lower statistics, these constraints becomes $<100 R_{\mathrm{g}}$, for the 3 last observations.

The presence of a soft X-ray excess, above the 3-10 keV power law extrapolation, is clearly visible in the first two observations and the addition of a multicolored disk component statistically improves the fits. This is not the case for the last three observations. The evolution of the disk component (decrease of its inner temperature and total flux) agrees with a disk recession from OBS1 $\left(R_{\text {in }} \sim 10 R_{\mathrm{g}}\right)$ to OBS2 $\left(R_{\text {in }} \sim 20 R_{\mathrm{g}}\right)$. However, again, due to the low statistics, we cannot confirm this recession for the last observations. A comparison with the very long Suzaku observation of GX 339-4 during a long faint hard state of its 2007-2008 outburst (T09), in a flux state similar to our OBS5, shows a relatively good agreement of the spectral shape between the two observations. The line shape is however inconsistent between the two pointings. In 2008 the source was observed during a persistent, but faint, hard state, while it was clearly in the decreasing phase of the outburst in 2011. It is then possible that the accretion flow had more time to evolve into a truncated accretion disk in 2008 compared to 2011.

The use of a model that includes blurred ionized reflection and thermal comptonization continuum gives better fits than the simple POWER LAW + DISKBB model used previously, at least for
OBS1 and OBS2. For the other observations both models give similar results. This model gives constraints for $R_{\text {in }}$ in marginal agreement with a disk recession. Hints of this recession also come from the increase of the $l_{\mathrm{h}} / l_{\mathrm{s}}$ compacity ratio of the hot corona along all the monitoring, and from the deviation of the disk flux to the Stefan law in $T^{4}$. These hints have to be taken with care, however, given the low statistics and the complexity of the model, this may lead to strong degeneracy between parameters.

Finally, signatures of ionized absorption seem to be present in at least OBS1 but absent in the other observations. With the radio re-ignition that occurs in between OBS1 and OBS2 (see C13), we suggest that the accretion flow may have transited from a disk wind, which is an ubiquitous characteristic of soft states, and a jet, which is signature of the hard states, during these two observations,. These absorption features may be the last signature of the disk wind before the transition to a jet-dominated state.

Acknowledgements. The authors thank the referee for a careful reading of the manuscript and for his/her comments that have significantly improved its quality. P.O.P. acknowledges financial support from CNES. This work is part of the CHAOS project ANR-12-BS05-0009 supported by the French Research National Agency (http: //www. chaos-project.fr). This research has made use of data obtained from the Suzaku satellite, a collaborative mission between the space agencies of Japan (JAXA) and the USA (NASA).

\section{References}

Barret, D., Olive, J. F., Boirin, L., et al. 2000, ApJ, 533, 329

Belloni, T., Homan, J., Casella, P., et al. 2005, A\&A, 440, 207

Blandford, R. D., \& Königl, A. 1979, ApJ, 232, 34

Buxton, M. M., Bailyn, C. D., Capelo, H. L., et al. 2012, AJ, 143, 130

Cabanac, C., Fender, R. P., Dunn, R. J. H., \& Koerding, E. G. 2009, MNRAS, 396,1415

Cadolle Bel, M., Rodriguez, J., D’Avanzo, P., et al. 2011, A\&A, 534, A119

Coppi, P. S. 1999, in High Energy Processes in Accreting Black Holes, eds. J. Poutanen, \& R. Svensson, ASP Conf. Ser., 161, 375

Corbel, S., Fender, R. P., Tzioumis, A. K., et al. 2000, A\&A, 359, 251

Corbel, S., Nowak, M. A., Fender, R. P., Tzioumis, A. K., \& Markoff, S. 2003, A\&A, 400, 1007

Corbel, S., Fender, R. P., Tomsick, J. A., Tzioumis, A. K., \& Tingay, S. 2004, ApJ, 617, 1272

Corbel, S., Aussel, H., Broderick, J. W., et al. 2013, MNRAS, 431, L107

Coriat, M., Corbel, S., Prat, L., et al. 2011, MNRAS, 414, 677

Davis, S. W., Done, C., \& Blaes, O. M. 2006, ApJ, 647, 525

Dhawan, V., Mirabel, I. F., \& Rodríguez, L. F. 2000, ApJ, 543, 373

Diaz Trigo, M., \& Boirin, L. 2013, Acta Polytechnica, 53, Supplement, 659

Diaz Trigo, M., Miller-Jones, J., Migliari, S., Parmar, A., \& Boirin, L.

2011, in The X-ray Universe 2011, eds. J.-U. Ness, \& M. Ehle, 3 available at http://xmm.esac.esa.int/external/xmm_science/ workshops/2011symposium/

Díaz Trigo, M., Sidoli, L., Boirin, L., \& Parmar, A. N. 2012, A\&A, 543, A50

Dinçer, T., Kalemci, E., Buxton, M. M., et al. 2012, ApJ, 753, 55

Done, C., \& Diaz Trigo, M. 2010, MNRAS, 407, 2287

Done, C., Gierlinski, M., \& Kubota, A. 2007, A\&ARv, 15, 1

Done, C., Davis, S. W., Jin, C., Blaes, O., \& Ward, M. 2012, MNRAS, 420, 1848

Dove, J. B., Wilms, J., Maisack, M., \& Begelman, M. C. 1997, ApJ, 487, 759

Dunn, R. J. H., Fender, R. P., Körding, E. G., Belloni, T., \& Cabanac, C. 2010, MNRAS, 403, 61

Esin, A. A., McClintock, J. E., \& Narayan, R. 1997, ApJ, 489, 865

Fabian, A. C., Parker, M. L., Wilkins, D. R., et al. 2014, MNRAS, in press [arXiv: 1401.1615]

Fender, R., Corbel, S., Tzioumis, T., et al. 1999, ApJ, 519, L165

Fender, R., Wu, K., Johnston, H., et al. 2004, Nature, 427, 222

Ferreira, J., Petrucci, P.-O., Henri, G., Sauge, L., \& Pelletier, G. 2006, A\&A, 447,813

Gallo, E., Fender, R. P., \& Pooley, G. G. 2003, MNRAS, 344, 60

Gierlinski, M., Zdziarski, A. A., Done, C., et al. 1997, MNRAS, 288, 958

Joinet, A., Jourdain, E., Malzac, J., et al. 2007, ApJ, 657, 400

Juett, A. M., Schulz, N. S., Chakrabarty, D., \& Gorczyca, T. W. 2006, ApJ, 648 , 1066

Kalemci, E., Dinçer, T., Tomsick, J. A., et al. 2013, ApJ, 779, 95 
Kong, A. K. H. 2008, ATel, 1588, 1

Kubota, A., Tanaka, Y., Makishima, K., et al. 1998, PASJ, 50, 667 Laor, A. 1991, ApJ, 376, 90

Luo, Y., \& Fang, T. 2014, ApJ, 780, 170

Malzac, J., Beloborodov, A. M., \& Poutanen, J. 2001, MNRAS, 326, 417

Merloni, A., Fabian, A. C., \& Ross, R. R. 2000, MNRAS, 313, 193

Meyer, F., Liu, B. F., \& Meyer-Hofmeister, E. 2000, A\&A, 354, L67

Miller, J. M., Ballantyne, D. R., Fabian, A. C., \& Lewin, W. H. G. 2002 MNRAS, 335, 865

Miller, J. M., Raymond, J., Fabian, A. C., et al. 2004, ApJ, 601, 450

Miller, J. M., Homan, J., \& Miniutti, G. 2006a, ApJ, 652, L113

Miller, J. M., Homan, J., Steeghs, D., et al. 2006b, ApJ, 653, 525

Miller, J. M., Raymond, J., Fabian, A., et al. 2006c, Nature, 441, 953

Miller, J. M., Raymond, J., Homan, J., et al. 2006d, ApJ, 646, 394

Neilsen, J., \& Lee, J. C. 2009, Nature, 458, 481

Petrucci, P.-O., Ferreira, J., Henri, G., \& Pelletier, G. 2008, MNRAS, 385, L88

Plant, D. S., Fender, R. P., Ponti, G., Munoz-Darias, T., \& Coriat, M. 2013,

MNRAS, submitted [arXiv: 1309.4781]

Ponti, G., Fender, R. P., Begelman, M. C., et al. 2012, MNRAS, L417

Poutanen, J., Krolik, J. H., \& Ryde, F. 1997, MNRAS, 292, L21

Protassov, R., van Dyk, D. A., Connors, A., Kashyap, V. L., \& Siemiginowska, A. 2002, ApJ, 571, 545
Reis, R. C., Fabian, A. C., Ross, R. R., et al. 2008, MNRAS, 387, 1489

Remillard, R. A., \& McClintock, J. E. 2006, ARA\&A, 44, 49

Reynolds, M. T., \& Miller, J. M. 2013, ApJ, 769, 16

Ross, R. R., \& Fabian, A. C. 2005, MNRAS, 358, 211

Russell, D. M., Altamirano, D., Lewis, F., et al. 2008, ATel, 1586, 1

Rykoff, E. S., Miller, J. M., Steeghs, D., \& Torres, M. A. P. 2007, ApJ, 666, 1129

Salvesen, G., Miller, J. M., Reis, R. C., \& Begelman, M. C. 2013, MNRAS, 431, 3510

Shimura, T., \& Takahara, F. 1995, ApJ, 445, 780

Sobczak, G. J., McClintock, J. E., Remillard, R. A., et al. 1999, ApJ, 517, L121

Sobolewska, M. A., Papadakis, I. E., Done, C., \& Malzac, J. 2011, MNRAS, 417,280

Stirling, A. M., Spencer, R. E., de la Force, C. J., et al. 2001, MNRAS, 327, 1273

Tomsick, J. A., Yamaoka, K., Corbel, S., et al. 2009, ApJ, 707, L87

Wilms, J., Allen, A., \& McCray, R. 2000, ApJ, 542, 914

Zdziarski, A. A., Gierlinski, M., Mikotajewska, J., et al. 2004, MNRAS, 351, 791

Zimmerman, E. R., Narayan, R., McClintock, J. E., \& Miller, J. M. 2005, ApJ 618,832

Zycki, P. T., Done, C., \& Smith, D. A. 1998, ApJ, 496, L25 